\title{
A REDUCED HESSIAN METHOD FOR LARGE-SCALE CONSTRAINED OPTIMIZATION
}

\author{
by
}

Lorenz T. Biegler, ${ }^{1}$ Jorge Nocedal, ${ }^{2}$ and Claudia Schmid ${ }^{1}$

\begin{abstract}
${ }^{1}$ Chemical Engineering Department, Carnegie Mellon University, Pittsburgh, PA 15213. Partial support for these authors is acknowledged from the Engineering Design Research Center, an NSF-sponsored Engineering Research Center at Carnegie Mellon University.

2 Department of Electrical Engineering and Computer Science, Northwestern University, Evanston IL 60208, nocedal@eecs.nwu.edu; also, Mathematics and Computer Science Division, Argonne National Laboratory, Argonne, IL 60439. This author was supported by National Science Foundation grants CCR9101359 and ASC-9213149, by U.S. Department of Energy grant DE-FG02-87ER25047-A004, and by the Office of Scientific Computing, U.S. Department of Energy, under Contract W-31-109-Eng-38.
\end{abstract}




\title{
A REDUCED HESSIAN METHOD FOR LARGE-SCALE CONSTRAINED OPTIMIZATION
}

\author{
by \\ Lorenz T. Biegler, Jorge Nocedal, and Claudia Schmid
}

\begin{abstract}
We propose a quasi-Newton algorithm for solving large optimization problems with nonlinear equality constraints. It is designed for problems with few degrees of freedom and is motivated by the need to use sparse matrix factorizations. The algorithm incorporates a correction vector that approximates the cross term $Z^{T} W Y p_{\mathrm{Y}}$ in order to estimate the curvature in both the range and null spaces of the constraints. The algorithm can be considered to be, in some sense, a practical implementation of an algorithm of Coleman and Conn. We give conditions under which local and superlinear convergence is obtained.
\end{abstract}

Key words: successive quadratic programming, reduced Hessian methods, constrained optimization, quasi-Newton method, large-scale optimization.

Abbreviated title: A Reduced Hessian Method

\section{Introduction}

We consider the nonlinear optimization problem

$$
\begin{gathered}
\min _{x \in \mathbf{R}^{n}} f(x) \\
\text { subject to } c(x)=0,
\end{gathered}
$$

where $f: \mathbf{R}^{n} \rightarrow \mathbf{R}$ and $c: \mathbf{R}^{n} \rightarrow \mathbf{R}^{m}$ are smooth functions. We are particularly interested in the case when the number of variables $n$ is large, and the algorithm we propose, which is a variation of the successive quadratic programming method, is designed to be efficient in this case. We assume that the first derivatives of $f$ and $c$ are available, but our algorithm does not require second derivatives.

The successive quadratic programming (SQP) method for solving (1.1)-(1.2) generates, at an iterate $x_{k}$, a search direction $d_{k}$ by solving

$$
\begin{gathered}
\min _{d \in \mathbf{R}^{n}} g\left(x_{k}\right)^{T} d+\frac{1}{2} d^{T} W\left(x_{k}\right) d \\
\text { subject to } c\left(x_{k}\right)+A\left(x_{k}\right)^{T} d=0,
\end{gathered}
$$


where $g$ denotes the gradient of $f, W$ denotes the Hessian of the Lagrangian function $L(x, \lambda)=$ $f(x)+\lambda^{T} c(x)$, and $A$ denotes the $n \times m$ matrix of constraint gradients

$$
A(x)=\left[\nabla c_{1}(x), \ldots, \nabla c_{m}(x)\right] .
$$

A new iterate is then computed as

$$
x_{k+1}=x_{k}+\alpha_{k} d_{k},
$$

where $\alpha_{k}$ is a steplength parameter chosen so as to reduce the value of the merit function. In this study we will use the $\ell_{1}$ merit function

$$
\phi_{\mu}(x)=f(x)+\mu\|c(x)\|_{1},
$$

where $\mu$ is a penalty parameter; see, for example, Conn (1973), Han (1977) or Fletcher (1987). We could have used other merit functions, but the essential points we wish to convey in this article are not dependent upon the particular choice of the merit function.

The solution of the quadratic program (1.3)-(1.4) can be written in a simple form if we choose a suitable basis of $\mathbf{R}^{n}$ to represent the search direction $d_{k}$. For this purpose, we introduce a nonsingular matrix of dimension $n$, which we write as

$$
\left[\begin{array}{ll}
Y_{k} & Z_{k}
\end{array}\right]
$$

where $Y_{k} \in \mathbf{R}^{n \times m}$ and $Z_{k} \in \mathbf{R}^{n \times(n-m)}$, and we assume that

$$
A_{k}^{T} Z_{k}=0 .
$$

(From now on we abbreviate $A\left(x_{k}\right)$ as $A_{k}, g\left(x_{k}\right)$ as $g_{k}$, etc.) Thus $Z_{k}$ is a basis for the tangent space of the constraints. We can now express $d_{k}$, the solution to (1.3)-(1.4), as

$$
d_{k}=Y_{k} p_{\mathrm{Y}}+Z_{k} p_{\mathrm{Z}}
$$

for some vectors $p_{\mathrm{Y}} \in \mathbf{R}^{m}$ and $p_{\mathrm{Z}} \in \mathbf{R}^{n-m}$. Due to (1.9) the linear constraints (1.4) become

$$
c_{k}+A_{k}^{T} Y_{k} p_{\mathrm{Y}}=0 .
$$

If we assume that $A_{k}$ has full column rank, then the nonsingularity of $\left[Y_{k} Z_{k}\right]$ and equation (1.9) imply that the matrix $A_{k}^{T} Y_{k}$ is nonsingular, so that $p_{\mathrm{Y}}$ is determined by (1.11):

$$
p_{\mathrm{Y}}=-\left[A_{k}^{T} Y_{k}\right]^{-1} c_{k} \text {. }
$$

Substituting this in (1.10), we have

$$
d_{k}=-Y_{k}\left[A_{k}^{T} Y_{k}\right]^{-1} c_{k}+Z_{k} p_{\mathrm{Z}}
$$

Note that

$$
Y_{k}\left[A_{k}^{T} Y_{k}\right]^{-1}
$$

is a right inverse of $A_{k}^{T}$ and that the first term in (1.13) represents a particular solution of the linear equations (1.4).

We have thus reduced the size of the SQP subproblem, which can now be expressed exclusively in terms of the variables $p_{\mathrm{Z}}$. Indeed, substituting (1.10) into (1.3), considering $Y_{k} p_{\mathrm{Y}}$ as constant, and ignoring constant terms, we obtain the unconstrained quadratic problem

$$
\min _{p_{\mathrm{Z}} \in \mathbf{R}^{n-m}}\left(Z_{k}^{T} g_{k}+Z_{k}^{T} W_{k} Y_{k} p_{\mathrm{Y}}\right)^{T} p_{\mathrm{Z}}+\frac{1}{2} p_{\mathrm{Z}}^{T}\left(Z_{k}^{T} W_{k} Z_{k}\right) p_{\mathrm{Z}}
$$


If we assume that $Z_{k}^{T} W_{k} Z_{k}$ is positive definite, the solution of (1.15) is

$$
p_{\mathrm{Z}}=-\left(Z_{k}^{T} W_{k} Z_{k}\right)^{-1}\left[Z_{k}^{T} g_{k}+Z_{k}^{T} W_{k} Y_{k} p_{\mathrm{Y}}\right]
$$

This determines the search direction of the SQP method.

We are particularly interested in the class of problems in which the number of variables $n$ is large, but $n-m$ is small. In this case it is practical to approximate $Z_{k}^{T} W_{k} Z_{k}$ using a variable metric formula such as BFGS. On the other hand, the matrix $Z_{k}^{T} W_{k} Y_{k}$, of dimension $(n-m) \times m$ may be too expensive to compute directly when $m$ is large. For this reason several authors simply ignore the "cross term" $Z_{k}^{T} W_{k} Y_{k} p_{\mathrm{Y}}$ in (1.16) and compute only an approximation to the reduced Hessian $Z_{k}^{T} W_{k} Z_{k}$; see Coleman and Conn (1984), Nocedal and Overton (1985), and Xie (1991). This approach is quite adequate when the basis matrices $Y_{k}$ and $Z_{k}$ in (1.8) are chosen to be orthonormal (Gurwitz and Overton (1989)).

For large problems, however, computing orthogonal bases can be expensive, and it is more efficient to obtain $Y_{k}$ and $Z_{k}$ by simple elimination of variables (cf. Fletcher (1987)). Unfortunately, in this case ignoring the cross term $Z_{k}^{T} W_{k} Y_{k} p_{\mathrm{Y}}$ can make the algorithm inefficient, as is illustrated by an example given in a companion paper (Biegler, Nocedal, and Schmid (1993)). The central point is that the range space component $Y_{k} p_{\mathrm{Y}}$ may be very large, and ignoring the contribution from the cross term in (1.16) can result in a poor step.

Therefore, in this paper we suggest ways of approximating the cross term $Z_{k}^{T} W_{k} Y_{k} p_{\mathrm{Y}}$ by a vector $w_{k}$,

$$
\left[Z_{k}^{T} W_{k} Y_{k}\right] p_{\mathrm{Y}} \approx w_{k}
$$

without computing the matrix $Z_{k}^{T} W_{k} Y_{k}$. We consider two approaches for calculating $w_{k}$; the first involves an approximation to the matrix $\left[Z_{k}^{T} W_{k} Y_{k}\right]$ using Broyden's update, and the second generates $w_{k}$ using finite differences. We will show that the rate of convergence of the new algorithm is 1-step Q-superlinear, as opposed to the 2-step superlinear rate for methods that ignore the cross term (Byrd (1985) and Yuan (1985)). The null space step (1.16) of our algorithm will be given by

$$
p_{\mathrm{Z}}=-\left(Z_{k}^{T} W_{k} Z_{k}\right)^{-1}\left[Z_{k}^{T} g_{k}+\zeta_{k} w_{k}\right]
$$

where $0<\zeta_{k} \leq 1$ is a damping factor to be discussed later on.

To describe our first strategy for computing the vector $w_{k}$, we consider a quasi-Newton method in which the rectangular matrix $Z_{k}^{T} W_{k}$ is approximated by a matrix $S_{k}$, using Broyden's method. We then obtain $w_{k}$ by multiplying this matrix by $Y_{k} p_{\mathrm{Y}}$, that is,

$$
w_{k}=S_{k} Y_{k} p_{\mathrm{Y}}
$$

How should $S_{k}$ be updated? Since $W_{k+1}=\nabla_{x x}^{2} L\left(x_{k+1}, \lambda_{k+1}\right)$, we have that

$$
Z_{k}^{T} W_{k+1}\left(x_{k+1}-x_{k}\right) \approx Z_{k}^{T}\left[\nabla_{x} L\left(x_{k+1}, \lambda_{k+1}\right)-\nabla_{x} L\left(x_{k}, \lambda_{k+1}\right)\right]
$$

when $x_{k+1}$ is close to $x_{k}$. We use this relation to establish the following secant equation: we demand that $S_{k+1}$ satisfy

$$
S_{k+1}\left(x_{k+1}-x_{k}\right)=Z_{k}^{T}\left[\nabla_{x} L\left(x_{k+1}, \lambda_{k+1}\right)-\nabla_{x} L\left(x_{k}, \lambda_{k+1}\right)\right] .
$$

One point in this derivation requires clarification. In the left-hand side of (1.19) we have $Z_{k}^{T} W_{k+1}$, and not $Z_{k+1}^{T} W_{k+1}$. We could have used $Z_{k+1}$ in (1.19), avoiding an inconsistency of indices, but this is not necessary since we will show that using $Z_{k}$ instead of $Z_{k+1}$ in (1.20) results in algorithms with all the desirable properties. This fact will not be surprising to readers familiar 
with the analysis of SQP methods; see, for example, Coleman and Conn (1984) or Nocedal and Overton (1985). In addition, using $Z_{k}$ allows updating of $S_{k+1}$ and $B_{k+1}$ prior to creating $Z_{k+1}$ at the new point.

Let us now consider how to approximate the reduced Hessian matrix $Z_{k}^{T} W_{k} Z_{k}$. Using (1.6) and (1.10) in (1.20), we obtain

$$
\left[S_{k+1} Z_{k}\right] \alpha_{k} p_{\mathrm{Z}}=-\alpha_{k} S_{k+1}\left(Y_{k} p_{\mathrm{Y}}\right)+Z_{k}^{T}\left[\nabla_{x} L\left(x_{k+1}, \lambda_{k+1}\right)-\nabla_{x} L\left(x_{k}, \lambda_{k+1}\right)\right] .
$$

Since $S_{k+1}$ approximates $Z_{k}^{T} W_{k}$, this suggests the following secant equation for $B_{k+1}$, the quasiNewton approximation to the reduced Hessian $Z_{k}^{T} W_{k} Z_{k}$ :

$$
B_{k+1} s_{k}=y_{k},
$$

where $s_{k}$ is defined by

$$
s_{k}=\alpha_{k} p_{\mathrm{Z}}
$$

and $y_{k}$ by

$$
y_{k}=Z_{k}^{T}\left[\nabla_{x} L\left(x_{k+1}, \lambda_{k+1}\right)-\nabla_{x} L\left(x_{k}, \lambda_{k+1}\right)\right]-\bar{w}_{k},
$$

with

$$
\bar{w}_{k}=\alpha_{k} S_{k+1}\left(Y_{k} p_{\mathrm{Y}}\right) .
$$

We will update $B_{k}$ by the BFGS formula (cf. Fletcher (1987))

$$
B_{k+1}=B_{k}-\frac{B_{k} s_{k} s_{k}^{T} B_{k}}{s_{k}^{T} B_{k} s_{k}}+\frac{y_{k} y_{k}^{T}}{y_{k}^{T} s_{k}}
$$

provided $s_{k}^{T} y_{k}$ is sufficiently positive.

We highlight a subtle but important point. We have defined two correction terms, $w_{k}$ and $\bar{w}_{k}$. Both are approximations to the cross term $\left(Z^{T} W Y\right) p_{\mathrm{Y}}$. The first term, $w_{k}$, which is needed to define the null-space step (1.18) - and thus the new iterate $x_{k+1}$ - makes use of the matrix $S_{k}$. The second term, $\bar{w}_{k}$, which is used in (1.22) to define the BFGS update of $B_{k}$, is computed by using the new Broyden matrix $S_{k+1}$ and takes into account the steplength $\alpha_{k}$. We will see below that it is useful to incorporate the most recent information in $\bar{w}_{k}$. Note that this requires the Broyden update to be applied before the vector $y_{k}$ for the BFGS update can be calculated from (1.22).

The Lagrange multiplier estimates $\lambda_{k}$ needed in the definition (1.22) of $y_{k}$ are defined by

$$
\lambda_{k}=-\left[Y_{k}^{T} A_{k}\right]^{-1} Y_{k}^{T} g_{k} .
$$

This formula is motivated by the fact that, at a solution $x_{*}$ of (1.1)-(1.2), we have $-g_{*}=A_{*} \lambda_{*}$, and since $Y_{*}\left[A_{*}^{T} Y_{*}\right]^{-1}$ is a right inverse of $A_{*}^{T}$,

$$
\lambda_{*}=-\left[Y_{*}^{T} A_{*}\right]^{-1} Y_{*}^{T} g_{*} .
$$

Using the same right inverse (1.14) in the definitions of $p_{\mathrm{Y}}$ and $\lambda_{k}$ will allow us a convenient simplification in the formulae presented in the following sections. We stress, however, that other Lagrange multiplier estimates can be used and that the best choice in practice might be the one that involves the least computation or storage.

We can now outline the sequential quadratic programming method analyzed in this paper.

\section{Algorithm I}


1. Choose constants $\eta \in(0,1 / 2)$ and $\tau, \tau^{\prime}$ with $0<\tau<\tau^{\prime}<1$. Set $k:=1$, and choose a starting point $x_{1}$ and an $(n-m) \times(n-m)$ symmetric and positive definite starting matrix $B_{1}$.

2. Evaluate $f_{k}, g_{k}, c_{k}$, and $A_{k}$, and compute $Y_{k}$ and $Z_{k}$.

3. Compute $p_{\mathrm{Y}}$ by solving the system

$$
\left(A_{k}^{T} Y_{k}\right) p_{\mathrm{Y}}=-c_{k} . \quad \text { (range space step) }
$$

4. Compute an approximation $w_{k}$ to $\left(Z_{k}^{T} W_{k} Y_{k}\right) p_{\mathrm{Y}}$.

5. Choose the damping parameter $\zeta_{k} \in(0,1]$ and compute $p_{\mathrm{Z}}$ from

$$
B_{k} p_{\mathrm{Z}}=-\left[Z_{k}^{T} g_{k}+\zeta_{k} w_{k}\right] . \quad \text { (null space step) }
$$

Define the search direction by

$$
d_{k}=Y_{k} p_{\mathrm{Y}}+Z_{k} p_{\mathrm{Z}}
$$

6. Set $\alpha_{k}=1$, and choose the weight $\mu_{k}$ of the merit function (1.7).

7. Test the line search condition

$$
\phi_{\mu_{k}}\left(x_{k}+\alpha_{k} d_{k}\right) \leq \phi_{\mu_{k}}\left(x_{k}\right)+\eta \alpha_{k} D \phi_{\mu_{k}}\left(x_{k} ; d_{k}\right),
$$

where $D \phi_{\mu_{k}}\left(x_{k} ; d_{k}\right)$ is the directional derivative of the merit function $\phi$ in the direction $d_{k}$.

8. If (1.29) is not satisfied, choose a new $\alpha_{k} \in\left[\tau \alpha_{k}, \tau^{\prime} \alpha_{k}\right]$ and go to (7); otherwise set

$$
x_{k+1}=x_{k}+\alpha_{k} d_{k} .
$$

9. Evaluate $f_{k+1}, g_{k+1}, c_{k+1}$, and $A_{k+1}$, and compute $Y_{k+1}$ and $Z_{k+1}$.

10. Compute the Lagrange multiplier estimate

$$
\lambda_{k+1}=-\left[Y_{k+1}^{T} A_{k+1}\right]^{-1} Y_{k+1}^{T} g_{k+1} .
$$

Define $\bar{w}_{k}$ (as will be discussed in $\S 3$ ), and compute

$$
s_{k}=\alpha_{k} p_{\mathrm{Z}}
$$

and

$$
y_{k}=Z_{k}^{T}\left[\nabla_{x} L\left(x_{k+1}, \lambda_{k+1}\right)-\nabla_{x} L\left(x_{k}, \lambda_{k+1}\right)\right]-\bar{w}_{k} .
$$

If the update criterion (to be discussed in $\S 3.3$ ) is satisfied, compute $B_{k+1}$ by the BFGS formula (1.24); else set $B_{k+1}=B_{k}$.

11. Set $k:=k+1$, and go to (3).

The algorithm has been left in a very general form, but in the next sections we discuss all its aspects in detail. In $\S 2$ we consider the choice of the basis matrices $Y_{k}$ and $Z_{k}$. In $\S 3$ we describe the calculation of the correction terms $w_{k}$ and $\bar{w}_{k}$, the conditions under which BFGS updating takes place, the choice of the damping parameter $\zeta_{k}$, and the procedure for updating the weight $\mu_{k}$ in the merit function. In $\S 4$ and $\S 5$ we analyze of the local behavior of the algorithm and 
show that the rate of convergence is at least R-linear. In $\S 6$ we present a superlinear convergence result, and some final remarks in $\S 7$ conclude the paper.

We now make a few comments about our notation. Throughout the paper, the vectors $p_{\mathrm{Y}}$ and $p_{\mathrm{Z}}$ are computed at $x_{k}$ and could be denoted by $p_{\mathrm{Y}}{ }^{(k)}$ and $p_{\mathrm{Z}}{ }^{(k)}$, but we will normally omit the superscript for simplicity. The symbol $\|\cdot\|$ denotes the $l_{2}$ vector norm or the corresponding induced matrix norm. When using the $l_{1}$ or $l_{\infty}$ norms we will indicate it explicitly by writing $\|\cdot\|_{1}$ or $\|\cdot\|_{\infty}$. A solution of problem (1.1) is denoted by $x_{*}$, and we define

$$
e_{k}=x_{k}-x_{*} \quad \text { and } \quad \sigma_{k}=\max \left\{\left\|e_{k}\right\|,\left\|e_{k+1}\right\|\right\} .
$$

Here, and for the rest of the paper, $\nabla L(x, \lambda)$ indicates the gradient of the Lagrangian with respect to $x$ only.

\section{The Basis Matrices}

As long as $Z_{k}$ spans the null space of $A_{k}^{T}$, and $\left[Y_{k} Z_{k}\right]$ is nonsingular, the choice of $Y_{k}$ and $Z_{k}$ is arbitrary. However, from the viewpoint of numerical stability and robustness of the algorithm it is desirable to define $Y_{k}$ and $Z_{k}$ to be orthonormal, that is,

$$
\begin{aligned}
Z(x)^{T} Z(x) & =I_{n-m} \\
Y(x)^{T} Y(x) & =I_{m} \\
Y(x)^{T} Z(x) & =0 .
\end{aligned}
$$

One way of obtaining these matrices is by forming the QR factorization of $A$. For large problems, however, computing this QR factorization is often too expensive. Therefore many researchers, including Gabay (1982), Gilbert (1991), Fletcher (1987), Murray and Prieto (1992), and Xie (1991), consider other, nonorthogonal choices of $Y$ and $Z$. For example, if we partition $x$ into $m$ basic or dependent variables (which without loss of generality are assumed to be the first $m$ variables) and $n-m$ nonbasic or control variables, we induce the partition

$$
A(x)^{T}=[C(x) N(x)],
$$

where the $m \times m$ basis matrix $C(x)$ is assumed to be nonsingular. We now define $Z(x)$ and $Y(x)$ to be

$$
Z(x)=\left[\begin{array}{c}
-C(x)^{-1} N(x) \\
I
\end{array}\right] Y(x)=\left[\begin{array}{l}
I \\
0
\end{array}\right] .
$$

When $A(x)$ is large and sparse, a sparse LU decomposition of $C(x)$ can often be computed efficiently, and this approach will be considerably less expensive than the QR factorization of $A$. Note that from the assumed nonsingularity of $C(x)$ both $Y(x)$ and $Z(x)$ vary smoothly with $x$, provided the same partition of the variables is maintained. In our implementation of the new algorithm (Biegler, Nocedal, and Schmid (1993)) we choose $Y_{k}$ and $Z_{k}$ by (2.2).

There is a price to pay for using nonorthogonal bases. If the matrix $C$ is ill conditioned (and this can be difficult to detect), the step computation may be inaccurate. Moreover, even if the basis is well conditioned, the range space step $Y_{k} p_{Y}$ can be large, and ignoring the cross term can cause serious difficulties. This phenomenon is illustrated in a two-dimensional example given by Biegler, Nocedal, and Schmid (1993). It is shown in that example that if the cross term $Z_{k}^{T} W_{k} Y_{k} p_{\mathrm{Y}}$ is ignored, the ratio $\left\|x_{k}+d_{k}\right\| /\left\|x_{k}\right\|$ can be arbitrarily large, even close to the solution. It is also shown that these inefficiencies disappear if the cross term is approximated as suggested in the following sections. 
In the rest of the paper we allow much freedom in the choice of the basis matrices. They can be given by (2.2), can be orthonormal, or can be chosen in other ways. The only restrictions we impose are that $A_{k}^{T} Z_{k}=0$ is satisfied, that the $n \times n$ matrix $\left[Y_{k} Z_{k}\right]$ is nonsingular and well conditioned, and that this matrix varies smoothly in a neighborhood of the solution.

\section{Further Details of the Algorithm}

In this section we consider how to calculate approximations $w_{k}$ and $\bar{w}_{k}$ to $\left(Z_{k}^{T} W_{k} Y_{k}\right) p_{\mathrm{Y}}$ to be used in the determination of the search direction $p_{\mathrm{Z}}$ and in updating $B_{k}$, respectively. We also discuss when to skip the BFGS update of the reduced Hessian approximation, as well as the selection of the damping factor $\zeta_{k}$ and the penalty parameter $\mu_{k}$.

To calculate approximations to $\left(Z^{T} W Y\right) p_{\mathrm{Y}}$, we propose two approaches. First, we consider a finite difference approximation to $Z_{k}^{T} W_{k}$ along the direction $Y_{k} p_{\mathrm{Y}}$. While this approach requires additional evaluations of reduced gradients at each iteration, it gives rise to a very good step. The second, more economical approach defines $w_{k}$ and $\bar{w}_{k}$ in terms of a Broyden approximation to $Z_{k}^{T} W_{k}$, as discussed in $\S 1$, and requires no additional function or gradient evaluations. Our algorithm will normally use this second approach, but as we will later see, it is sometimes necessary to use finite differences.

\subsection{Calculating $w_{k}$ and $\bar{w}_{k}$ through Finite Differences}

We first calculate the range space step $p_{\mathrm{Y}}$ at $x_{k}$ through Equation (1.26). Next we compute the reduced gradient of the Lagrangian at $x_{k}+Y_{k} p_{\mathrm{Y}}$ and define

$$
w_{k}=Z_{k}^{T}\left[\nabla L\left(x_{k}+Y_{k} p_{\mathrm{Y}}, \lambda_{k}\right)-\nabla L\left(x_{k}, \lambda_{k}\right)\right] .
$$

After the step to the new iterate $x_{k+1}$ has been taken, we define

$$
\bar{w}_{k}=Z_{k}^{T}\left[\nabla L\left(x_{k}+\alpha_{k} Y_{k} p_{\mathrm{Y}}, \lambda_{k+1}\right)-\nabla L\left(x_{k}, \lambda_{k+1}\right)\right],
$$

which requires a new evaluation of gradients if $\alpha_{k} \neq 1$. Thus, up to three evaluations of the objective function gradient may be required at each iteration.

We note that this finite-difference approach is very similar to the algorithm of Coleman and Conn $(1982,1984)$. Starting at a point $z_{k}$, the Coleman-Conn algorithm (with steplength $\alpha_{k}=1$ ) is given by

$$
\begin{gathered}
Z_{k} p_{\mathrm{Z}}=-Z\left(z_{k}\right) B_{k}^{-1} Z\left(z_{k}\right)^{T} g\left(z_{k}\right) \\
Y_{k} p_{\mathrm{Y}}=-Y\left(z_{k}\right)\left[A\left(z_{k}\right)^{T} Y\left(z_{k}\right)\right]^{-1} c\left(z_{k}+Z_{k} p_{\mathrm{Z}}\right) \\
z_{k+1}=z_{k}+Z_{k} p_{\mathrm{Z}}+Y_{k} p_{\mathrm{Y}} .
\end{gathered}
$$

Let us now consider Algorithm I, and to better illustrate its similarity with the Coleman and Conn method, let us assume that instead of $(3.1), w_{k}$ is defined by

$$
w_{k}=Z\left(x_{k}+Y_{k} p_{\mathrm{Y}}\right)^{T} g\left(x_{k}+Y_{k} p_{\mathrm{Y}}\right)-Z\left(x_{k}\right)^{T} g\left(x_{k}\right),
$$

which differs from (3.1) by terms of order $O\left(\left\|p_{\mathrm{Y}}\right\|\right)$. Then Algorithm I with $\alpha_{k}=1$ and $\zeta_{k}=1$ is given by

$$
Y_{k} p_{\mathrm{Y}}=-Y\left(x_{k}\right)\left[A\left(x_{k}\right)^{T} Y\left(x_{k}\right)\right]^{-1} c\left(x_{k}\right) .
$$




$$
\begin{aligned}
Z_{k} p_{\mathrm{Z}}= & -Z\left(x_{k}\right) B_{k}^{-1}\left[Z\left(x_{k}\right)^{T} g\left(x_{k}\right)+w_{k}\right] \\
= & -Z\left(x_{k}\right) B_{k}^{-1}\left[Z\left(x_{k}+Y_{k} p_{\mathrm{Y}}\right)^{T} g\left(x_{k}+Y_{k} p_{\mathrm{Y}}\right)\right] . \\
& x_{k+1}=x_{k}+Y_{k} p_{\mathrm{Y}}+Z_{k} p_{\mathrm{Z}} .
\end{aligned}
$$

The similarity between the two approaches is apparent in Figure 1, especially if we consider the intermediate points in the Coleman-Conn iteration to be the starting and final points, respectively.

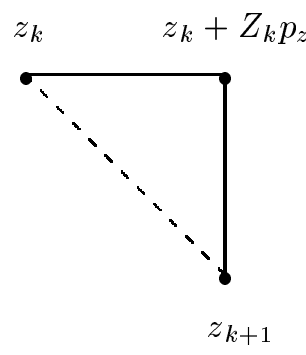

Coleman-Conn step

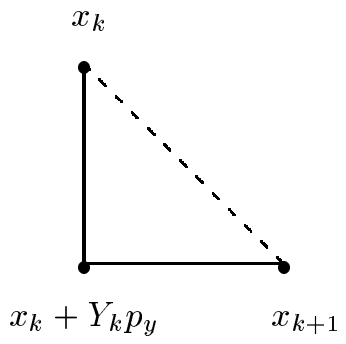

The step of Algorithm I

Figure 1

Comparison of Coleman-Conn method and Algorithm I

In the Coleman-Conn algorithm, the approximation $B_{k}$ to the reduced Hessian $Z_{k}^{T} W_{k} Z_{k}$ is obtained by moving along the null space direction $Z_{k} p_{Z}$, and making a new evaluation of the function and constraint gradients. To be more precise, Coleman and Conn define

$$
y_{k}=Z_{k}^{T}\left[\nabla L\left(x_{k}+Z_{k} p_{\mathrm{Z}}, \lambda_{k}\right)-\nabla L\left(x_{k}, \lambda_{k}\right)\right]
$$

and $s_{k}=Z_{k}^{T}\left[x_{k+1}-x_{k}\right]$ and apply a quasi-Newton formula to update $B_{k}$. Algorithm I, using finite differences, amounts essentially to the same thing. To see this, note that if Formula (3.2) is used in (1.33), then

$$
y_{k}=Z_{k}^{T}\left[\nabla L\left(x_{k+1}, \lambda_{k+1}\right)-\nabla L\left(x_{k}+\alpha_{k} Y_{k} p_{\mathrm{Y}}, \lambda_{k+1}\right)\right],
$$

which represents a difference in reduced gradients of the Lagrangian along the null space direction $Z_{k} p_{\mathrm{Z}}$.

Byrd (1990) and Gilbert (1989) showed that the sequence $\left\{z_{k}+Z_{k} p_{Z}\right\}$ (but not the sequence $\left\{z_{k}\right\}$ ) generated by the Coleman-Conn method converges one-step Q-superlinearly. If Algorithm I always computed the correction terms $w_{k}$ and $\bar{w}_{k}$ by finite differences, its cost and convergence behavior would be similar to those of the Coleman-Conn method (except when $\alpha_{k} \neq 1$, which requires one extra gradient evaluation for Algorithm I). However, we will often be able to avoid the use of finite differences and instead use the more economical approach discussed next.

\subsection{Using Broyden's Method to Compute $w_{k}$ and $\bar{w}_{k}$}

We can approximate the rectangular matrix $Z_{k}^{T} W_{k}$ by a matrix $S_{k}$ updated by Broyden's method, and then compute $w_{k}$ and $\bar{w}_{k}$ by post-multiplying this matrix by $Y_{k} p_{\mathrm{Y}}$ or by a multiple 
of this vector. As discussed in $\S 1$, it is reasonable to impose the secant equation (1.20) on this Broyden approximation, which can therefore be updated by the formula (cf. Fletcher (1987))

$$
S_{k+1}=S_{k}+\frac{\left(\bar{y}_{k}-S_{k} \bar{s}_{k}\right) \bar{s}_{k}^{T}}{\bar{s}_{k}^{T} \bar{s}_{k}}
$$

where

$$
\bar{y}_{k}=Z_{k}^{T}\left[\nabla L\left(x_{k+1}, \lambda_{k+1}\right)-\nabla L\left(x_{k}, \lambda_{k+1}\right)\right]
$$

and

$$
\bar{s}_{k}=x_{k+1}-x_{k}
$$

We now define

$$
w_{k}=S_{k} Y_{k} p_{\mathrm{Y}} \quad \text { and } \quad \bar{w}_{k}=\alpha_{k} S_{k+1} Y_{k} p_{\mathrm{Y}} .
$$

It should be noted that this approach requires the storage of the $(n-m) \times n$ matrix $S_{k}$, in addition to the reduced Hessian approximation, $B_{k}$. For problems where $n-m$ is small, this expense is far less than the storage of a full Hessian approximation to $W_{k}$. On the other hand, if $n-m$ is not very small, it may be preferable to use a limited-memory implementation of Broyden's method. Here the matrices $S_{k}$ are represented implicitly, using, for example, the compact representation described in Byrd, Nocedal, and Schnabel (1992). The advantage of the limited memory implementation is that it requires the storage of only a few $n$-vectors to represent $S$.

Since is no guarantee that the Broyden approximations $S_{k}$ will remain bounded, we need to safeguard them. At the beginning of the algorithm we choose a positive constant $\Gamma$ and define

$$
w_{k}:= \begin{cases}w_{k} & \text { if }\left\|w_{k}\right\| \leq \frac{\Gamma}{\left\|p_{\mathrm{Y}}\right\|^{1 / 2}}\left\|p_{\mathrm{Y}}\right\| \\ w_{k} \frac{\Gamma\left\|p_{\mathrm{Y}}\right\|^{1 / 2}}{\left\|w_{k}\right\|} & \text { otherwise. }\end{cases}
$$

The correction $\bar{w}_{k}$ will be safeguarded in a different way. We choose a sequence of positive numbers $\left\{\gamma_{k}\right\}$ such that $\Sigma_{k=1}^{\infty} \gamma_{k}<\infty$, and we set

$$
\bar{w}_{k}:= \begin{cases}\bar{w}_{k} & \text { if }\left\|\bar{w}_{k}\right\| \leq \alpha_{k}\left\|p_{\mathrm{Y}}\right\| / \gamma_{k} \\ \bar{w}_{k} \frac{\alpha_{k}\left\|p_{\mathrm{Y}}\right\|}{\gamma_{k}\left\|\bar{w}_{k}\right\|} & \text { otherwise. }\end{cases}
$$

As the iterates converge to the solution, $p_{\mathrm{Y}} \rightarrow 0$, so that from (3.12) and from the boundedness of $Y_{k}$ we see that these safeguards allow the Broyden updates $S_{k}$ to become unbounded, but in a controlled manner. We will show in $\S 4$ and $\S 5$ that with the safeguards (3.13) and (3.14) Algorithm I is locally and R-linearly convergent and that this implies that the Broyden updates $S_{k}$ do, in fact, remain bounded, so that the safeguards become inactive asymptotically.

Our Broyden approximation to the correction terms $w_{k}$ and $\bar{w}_{k}$ was motivated by recent work of Gurwitz (1993). She approximates $Z_{k}^{T} W_{k} Z_{k}$ by the BFGS formula with

$$
s_{k}=Z_{k}^{T}\left[x_{k+1}-x_{k}\right]
$$

and

$$
y_{k}=Z_{k}^{T}\left[\nabla L\left(x_{k+1}, \lambda_{k+1}\right)-\nabla L\left(x_{k}, \lambda_{k+1}\right)\right]
$$

and approximates $Z_{k}^{T} W_{k} Y_{k}$ by a matrix $D_{k}$ using Broyden's formula (3.9) with

$$
\bar{s}_{k}=Y_{k}^{T}\left[x_{k+1}-x_{k}\right]
$$




$$
\bar{y}_{k}=Z_{k}^{T}\left[\nabla L\left(x_{k+1}, \lambda_{k+1}\right)-\nabla L\left(x_{k}, \lambda_{k+1}\right)\right]-B_{k} p_{\mathrm{Z}} .
$$

Since the updates may not always be defined, Gurwitz proposes to sometimes skip the update of $B_{k}$ or $D_{k}$. She shows 1-step Q-superlinear convergence if and only if one of the updates is taken at each iteration, but this cannot be guaranteed. The analysis of this paper will show that it is preferable to update an approximation to $Z_{k}^{T} W_{k}$, as in Algorithm I, instead of an approximation to $Z_{k}^{T} W_{k} Y_{k}$, as proposed by Gurwitz, since our approach leads to 1 -step superlinear convergence in all cases.

A related method was derived by Coleman and Fenyes (1992). Their lower partition BFGS formula (LPB) simultaneously updates approximations to $Z_{k}^{T} W_{k} Z_{k}$ and $Z_{k}^{T} W_{k} Y_{k}$, by means of a new variational problem. The resulting updating formula requires the solution of a cubic equation, and its roots can correspond to cases where updates should be avoided (e.g., $s_{k}^{T} y_{k} \leq 0$ ). The drawback of this approach is that choosing the correct root is not always easy.

An earlier proposal by Tagliaferro (1989) consists of approximating the matrices $Z_{k}^{T} W_{k} Z_{k}$ and $Z_{k}^{T} W_{k} Y_{k}$ using the PSB update formula and Broyden's method, respectively. One disadvantage of this approach is that the matrices generated by this updating procedure may become very ill conditioned.

\subsection{Update Criterion}

It is well known that the BFGS update (1.24) is well defined only if the curvature condition $s_{k}^{T} y_{k}>0$ is satisfied. This condition can always be enforced in the unconstrained case by performing an appropriate line search; see, for example, Fletcher (1987). When constraints are present, however, the curvature condition $s_{k}^{T} y_{k}>0$ can be difficult to obtain, even near the solution.

To show this, we first note from (1.33), (1.28), and (1.32) and from the Mean Value theorem that

$$
\begin{aligned}
y_{k} & =Z_{k}^{T}\left[\int_{0}^{1} \nabla_{x x}^{2} L\left(x_{k}+\tau \alpha_{k} d_{k}, \lambda_{k+1}\right) d \tau\right] \alpha_{k} d_{k}-\bar{w}_{k} \\
& \equiv Z_{k}^{T} \tilde{W}_{k} \alpha_{k} d_{k}-\bar{w}_{k} \\
& =Z_{k}^{T} \tilde{W}_{k} Z_{k} s_{k}+\alpha_{k} Z_{k}^{T} \tilde{W}_{k} Y_{k} p_{\mathrm{Y}}-\bar{w}_{k},
\end{aligned}
$$

where we have defined

$$
\tilde{W}_{k}=\int_{0}^{1} \nabla_{x x}^{2} L\left(x_{k}+\tau \alpha_{k} d_{k}, \lambda_{k+1}\right) d \tau .
$$

Thus

$$
s_{k}^{T} y_{k}=s_{k}^{T}\left(Z_{k}^{T} \tilde{W}_{k} Z_{k}\right) s_{k}+\alpha_{k} s_{k}^{T}\left(Z_{k}^{T} \tilde{W}_{k} Y_{k}\right) p_{\mathrm{Y}}-s_{k}^{T} \bar{w}_{k} .
$$

Near the solution, the first term on the right-hand side will be positive, since $Z_{k}^{T} \tilde{W}_{k} Z_{k}$ can be assumed positive definite. Nevertheless, the last two terms are of uncertain sign and can make $s_{k}^{T} y_{k}$ negative. Several reduced Hessian methods in the literature set $\bar{w}_{k}$ equal to zero for all $k$, and update $B_{k}$ only if $p_{\mathrm{Y}}$ is small enough compared with $s_{k}$ that the first term in the right-hand side of (3.17) dominates the second term (see Nocedal and Overton (1985), Gurwitz and Overton (1989), and Xie (1991)).

Skipping the BFGS update may appear to be a crude heuristic, but we argue that it gives rise to a sound algorithm. First of all, the last two terms in (3.17) normally converge to zero faster than the first term, so that the right-hand side of (3.17) will often be positive near the solution and BFGS updating will take place frequently. Furthermore, if the right-hand side of (3.17) is 
negative, the range space step $Y_{k} p_{\mathrm{Y}}$ is relatively large, resulting in sufficient progress towards the solution. These arguments will be made more precise in $\S 5$.

We therefore opt for skipping the BFGS update, when necessary, and we now present a strategy for deciding when to do so. Recall that $\sigma_{k}$, defined by (1.34), converges to zero if the iterates converge to $x_{*}$.

\section{Update Criterion I}

Choose a constant $\gamma_{\mathrm{fd}}>0$ and a sequence of positive numbers $\left\{\gamma_{k}\right\}$ such that $\Sigma_{k=1}^{\infty} \gamma_{k}<\infty$ (this is the same sequence $\left\{\gamma_{k}\right\}$ that was used in (3.14)).

- If $\bar{w}_{k}$ is computed by Broyden's method, and if both $s_{k}^{T} y_{k}>0$ and

$$
\left\|p_{\mathrm{Y}}\right\| \leq \gamma_{k}^{2}\left\|p_{\mathrm{Z}}\right\|
$$

hold at iteration $k$, then update the matrix $B_{k}$ by means of the BFGS formula (1.24) with $s_{k}$ and $y_{k}$ given by (1.32) and (1.33). Otherwise, set $B_{k+1}=B_{k}$.

- If $\bar{w}_{k}$ is computed by finite differences, and if both $s_{k}^{T} y_{k}>0$ and

$$
\left\|p_{\mathrm{Y}}\right\| \leq \gamma_{\mathrm{fd}}\left\|p_{\mathrm{Z}}\right\| / \sigma_{k}^{1 / 2}
$$

hold at iteration $k$, then update the matrix $B_{k}$ by means of the BFGS formula (1.24) with $s_{k}$ and $y_{k}$ given by (1.32) and (1.33). Otherwise, set $B_{k+1}=B_{k}$.

Note that $\sigma_{k}$ requires knowledge of the solution vector $x_{*}$ and is therefore not computable. However, we will later see that $\sigma_{k}$ can be replaced by any quantity that is of the same order as the error $e_{k}$, for example, the optimality conditions $\left(\left\|Z_{k}^{T} g_{k}\right\|+\left\|c_{k}\right\|\right)$. Nevertheless, for convenience we will leave $\sigma_{k}$ in (3.19).

We now closely consider the properties of the BFGS matrices $B_{k}$ when Update Criterion I is used. Let us define

$$
\cos \theta_{k}=\frac{s_{k}^{T} B_{k} s_{k}}{\left\|s_{k}\right\|\left\|B_{k} s_{k}\right\|}
$$

which, as we will see, is a measure of the goodness of the null space step $Z_{k} p_{\mathrm{Z}}$. We begin by restating a theorem from Byrd and Nocedal (1989) regarding the behavior of $\cos \theta_{k}$ when the matrix $B_{k}$ is updated by the BFGS formula.

Theorem 3.1 Let $\left\{B_{k}\right\}$ be generated by the BFGS formula (1.24) where, for all $k \geq 1, s_{k} \neq 0$ and

$$
\begin{aligned}
\frac{y_{k}^{T} s_{k}}{s_{k}^{T} s_{k}} & \geq m>0 \\
\frac{\left\|y_{k}\right\|^{2}}{y_{k}^{T} s_{k}} & \leq M
\end{aligned}
$$

Then, there exist constants $\beta_{1}, \beta_{2}, \beta_{3}>0$ such that, for any $k \geq 1$, the relations

$$
\begin{aligned}
\cos \theta_{j} & \geq \beta_{1} \\
\beta_{2} \leq \frac{\left\|B_{j} s_{j}\right\|}{\left\|s_{j}\right\|} & \leq \beta_{3}
\end{aligned}
$$

hold for at least $\left\lceil\frac{1}{2} k\right\rceil$ values of $j \in[1, k]$. 
This theorem refers to the iterates for which BFGS updating takes place; but since, for the other iterates, $B_{k+1}=B_{k}$, the theorem characterizes the whole sequence of matrices $\left\{B_{k}\right\}$. Theorem 3.1 states that, if $s_{k}^{T} y_{k}$ is always sufficiently positive, in the sense that Conditions (3.21) and (3.22) are satisfied, then at least half of the iterates at which updating takes place are such that $\cos \theta_{j}$ is bounded away from zero and $B_{j} s_{j}=O\left(\left\|s_{j}\right\|\right)$. Since it will be useful to refer easily to these iterates, we make the following definition.

Definition 3.1 We define $J$ to be the set of iterates for which (3.23) and (3.24) hold. We call $J$ the set of "good iterates" and define $J_{k}=J \cap\{1,2, \ldots, k\}$.

Note that if the matrices $B_{k}$ are updated only a finite number of times, their condition number is bounded, and (3.23)-(3.24) are satisfied for all $k$. Thus in this case all iterates are good iterates.

We now study the case when BFGS updating takes place an infinite number of times. Let us assume that all functions under consideration are smooth and bounded. If at a solution point $x_{*}$ the reduced Hessian $Z_{*}^{T} W_{*} Z_{*}$ is positive definite, then for all $x_{k}$ in a neighborhood of $x_{*}$ the smallest eigenvalue of $Z_{k}^{T} \tilde{W}_{k} Z_{k}$ is bounded away from zero $\left(\tilde{W}_{k}\right.$ is defined in (3.16)). We now show that in such a neighborhood Update Criterion I implies (3.21)-(3.22).

Let us first consider the case when $\bar{w}_{k}$ is computed by Broyden's method. Using (3.17), (3.18), and (3.14), and since $\gamma_{k}$ converges to zero, we have

$$
\begin{aligned}
s_{k}^{T} y_{k} & \geq C\left\|s_{k}\right\|^{2}-O\left(\gamma_{k}^{2}\left\|s_{k}\right\|^{2}\right)-O\left(\gamma_{k}\left\|s_{k}\right\|^{2}\right) \\
& \geq m\left\|s_{k}\right\|^{2},
\end{aligned}
$$

for some positive constants $C, m$. Also, from (3.15), (3.18), and (3.14) we have that

$$
\begin{aligned}
\left\|y_{k}\right\| & \leq O\left(\left\|s_{k}\right\|\right)+O\left(\gamma_{k}^{2}\left\|s_{k}\right\|\right)+O\left(\gamma_{k}\left\|s_{k}\right\|\right) \\
& \leq O\left(\left\|s_{k}\right\|\right) .
\end{aligned}
$$

We thus see from (3.25)-(3.26) that there is a constant $M$ such that for all $k$ for which updating takes place,

$$
\frac{\left\|y_{k}\right\|^{2}}{y_{k}^{T} s_{k}} \leq M
$$

which together with (3.25) shows that (3.21)-(3.22) hold when Broyden's method is used.

If $\bar{w}_{k}$ is computed by the finite-difference formula (3.2), we see from (1.33) and the Mean Value theorem that there is a matrix $\hat{W}_{k}$ such that

$$
\begin{aligned}
y_{k} & =Z_{k}^{T}\left[\nabla L\left(x_{k+1}, \lambda_{k+1}\right)-\nabla L\left(x_{k}+\alpha_{k} Y_{k} p_{\mathrm{Y}}, \lambda_{k+1}\right)\right] \\
& \equiv Z_{k}^{T} \hat{W}_{k} Z_{k} s_{k} .
\end{aligned}
$$

Reasoning as before we see that (3.25) and (3.26) also hold in this case, and that (3.21)-(3.22) are satisfied in the case when finite differences are used. We have therefore established the following result.

Lemma 3.1 In a neighborhood of a solution point $x_{*}$, and whenever BFGS updating takes place as stipulated by Update Criterion I, $s_{k}^{T} y_{k}$ is sufficiently positive in the sense that (3.21)-(3.22) hold. 


\subsection{Choosing $\mu_{k}$ and $\zeta_{k}$}

We will now see that by appropriately choosing the penalty parameter $\mu_{k}$ and the damping parameter $\zeta_{k}$ for $w_{k}$, the search direction generated by Algorithm I is always a descent direction for the merit function. Moreover, for the good iterates $J$, it is a direction of strong descent.

Since $d_{k}$ satisfies the linearized constraint (1.11), it is easy to show (see Eq. (2.24) of Byrd and Nocedal (1991)) that the directional derivative of the $\ell_{1}$ merit function in the direction $d_{k}$ is given by

$$
D \phi_{\mu_{k}}\left(x_{k} ; d_{k}\right)=g_{k}^{T} d_{k}-\mu_{k}\left\|c_{k}\right\|_{1} .
$$

The fact that the same right inverse of $A_{k}^{T}$ is used in (1.26) and (1.31) implies that

$$
g_{k}^{T} Y_{k} p_{\mathrm{Y}}=\lambda_{k}^{T} c_{k} .
$$

Recalling the decomposition (1.28) and using (3.28), we obtain

$$
\begin{aligned}
D \phi_{\mu_{k}}\left(x_{k} ; d_{k}\right) & =g_{k}^{T} Z_{k} p_{\mathrm{Z}}-\mu_{k}\left\|c_{k}\right\|_{1}+\lambda_{k}^{T} c_{k} \\
& =\left(Z_{k}^{T} g_{k}+\zeta_{k} w_{k}\right)^{T} p_{\mathrm{Z}}-\zeta_{k} w_{k}^{T} p_{\mathrm{Z}}-\mu_{k}\left\|c_{k}\right\|_{1}+\lambda_{k}^{T} c_{k} .
\end{aligned}
$$

Now from (1.32) and (1.27) we have that

$$
B_{k} s_{k}=-\alpha_{k}\left(Z_{k}^{T} g_{k}+\zeta_{k} w_{k}\right) .
$$

Substituting this in (3.20), we obtain

$$
\cos \theta_{k}=\frac{-\left(Z_{k}^{T} g_{k}+\zeta_{k} w_{k}\right)^{T} p_{\mathrm{Z}}}{\left\|Z_{k}^{T} g_{k}+\zeta_{k} w_{k}\right\|\left\|p_{\mathrm{Z}}\right\|}
$$

Recalling the inequality $\lambda_{k}^{T} c_{k} \leq\left\|\lambda_{k}\right\|_{\infty}\left\|c_{k}\right\|_{1}$, and using (3.31) in (3.29), we obtain, for all $k$,

$$
D \phi_{\mu_{k}}\left(x_{k} ; d_{k}\right) \leq-\left\|Z_{k}^{T} g_{k}+\zeta_{k} w_{k}\right\|\left\|p_{\mathrm{Z}}\right\| \cos \theta_{k}-\zeta_{k} w_{k}^{T} p_{\mathrm{Z}}-\left(\mu_{k}-\left\|\lambda_{k}\right\|_{\infty}\right)\left\|c_{k}\right\|_{1} .
$$

Note also from (3.30) and (1.32) that

$$
\frac{\left\|s_{k}\right\|}{\left\|B_{k} s_{k}\right\|}=\frac{\left\|p_{\mathrm{z}}\right\|}{\left\|Z_{k}^{T} g_{k}+\zeta_{k} w_{k}\right\|}
$$

We now concentrate on the good iterates $J$, as given in Definition 3.1. If $j \in J$, we have from (3.33) and (3.24) that

$$
\frac{1}{\beta_{3}}\left\|Z_{j}^{T} g_{j}+\zeta_{j} w_{j}\right\| \leq\left\|p_{\mathrm{Z}}^{(j)}\right\| \leq \frac{1}{\beta_{2}}\left\|Z_{j}^{T} g_{j}+\zeta_{j} w_{j}\right\| .
$$

Using this and (3.23) in (3.32), we obtain, for $j \in J$,

$$
\begin{aligned}
D \phi_{\mu_{j}}\left(x_{j} ; d_{j}\right) & \leq-\frac{1}{\beta_{3}}\left\|Z_{j}^{T} g_{j}+\zeta_{j} w_{j}\right\|^{2} \cos \theta_{j}-\zeta_{k} w_{j}^{T} p_{\mathrm{Z}}^{(j)}-\left(\mu_{j}-\left\|\lambda_{j}\right\|_{\infty}\right)\left\|c_{j}\right\|_{1} \\
& \leq-\frac{\beta_{1}}{\beta_{3}}\left\|Z_{j}^{T} g_{j}\right\|^{2}-\frac{2 \zeta_{j} \cos \theta_{j}}{\beta_{3}}\left(g_{j}^{T} Z_{j} w_{j}\right)-\zeta_{j} w_{j}^{T} p_{\mathrm{Z}}^{(j)}-\left(\mu_{j}-\left\|\lambda_{j}\right\|_{\infty}\right)\left\|c_{j}\right\|_{1},
\end{aligned}
$$

where we have dropped the nonpositive term $-\zeta_{j}^{2} \cos \theta_{j}\left\|w_{j}\right\|^{2} / \beta_{3}$. Since we can assume that $\beta_{3}>1$ (it is defined as an upper bound in (3.24)), we have

$$
D \phi_{\mu_{j}}\left(x_{j} ; d_{j}\right) \leq-\frac{\beta_{1}}{\beta_{3}}\left\|Z_{j}^{T} g_{j}\right\|^{2}+\left[2 \zeta_{j} \cos \theta_{j}\left|g_{j}^{T} Z_{j} w_{j}\right|-\zeta_{j} w_{j}^{T} p_{Z}^{(j)}\right]-\left(\mu_{j}-\left\|\lambda_{j}\right\|_{\infty}\right)\left\|c_{j}\right\|_{1} .
$$


It is now clear that if

$$
2 \zeta_{j} \cos \theta_{j}\left|g_{j}^{T} Z_{j} w_{j}\right|-\zeta_{j} w_{j}^{T} p_{\mathrm{Z}}^{(j)} \leq \rho\left\|c_{j}\right\|_{1},
$$

for some constant $\rho$, and if

$$
\mu_{j} \geq\left\|\lambda_{j}\right\|_{\infty}+2 \rho
$$

then for all $j \in J$,

$$
D \phi_{\mu_{j}}\left(x_{j} ; d_{j}\right) \leq-\frac{\beta_{1}}{\beta_{3}}\left\|Z_{j}^{T} g_{j}\right\|^{2}-\rho\left\|c_{j}\right\|_{1} .
$$

This means that if (3.35) and (3.36) hold, then for the good iterates $j \in J$, the search direction $d_{j}$ is a strong direction of descent for the $\ell_{1}$ merit function in the sense that the first-order reduction is proportional to the KKT error.

We will choose $\zeta_{k}$ so that (3.35) holds for all iterations. To show how to do this, we note from (1.27) that

$$
p_{\mathrm{Z}}=-B_{k}^{-1} Z_{k}^{T} g_{k}-\zeta_{k} B_{k}^{-1} w_{k}
$$

so that, for $j=k,(3.35)$ can be written as

$$
\zeta_{k}\left[2 \cos \theta_{k}\left|g_{k}^{T} Z_{k} w_{k}\right|+w_{k}^{T} B_{k}^{-1} Z_{k}^{T} g_{k}+\zeta_{k} w_{k}^{T} B_{k}^{-1} w_{k}\right] \leq \rho\left\|c_{k}\right\|_{1} .
$$

Clearly this condition is satisfied for a sufficiently small and positive value of $\zeta_{k}$. Specifically, at the beginning of the algorithm we choose a constant $\rho>0$ and, at every iteration $k$, define

$$
\zeta_{k}=\min \left\{1, \hat{\zeta}_{k}\right\},
$$

where $\hat{\zeta_{k}}$ is the largest value that satisfies (3.38) as an equality.

The penalty parameter $\mu_{k}$ must satisfy (3.36), so we define it at every iteration of the algorithm by

$$
\mu_{k}= \begin{cases}\mu_{k-1} & \text { if } \mu_{k-1} \geq\left\|\lambda_{k}\right\|_{\infty}+2 \rho \\ \left\|\lambda_{k}\right\|_{\infty}+3 \rho & \text { otherwise. }\end{cases}
$$

The damping factor $\zeta_{k}$ and the updating formula for the penalty parameter $\mu_{k}$ have been defined so as to give strong descent for the good iterates $J$. We now show that they ensure that the search direction is also a direction of descent (but not necessarily of strong descent) for the other iterates, $k \notin J$. Since (3.35) holds for all iterations by our choice of $\zeta_{k}$, we have in particular

$$
-\zeta_{k} w_{k}^{T} p_{\mathrm{Z}} \leq \rho\left\|c_{k}\right\|_{1} .
$$

Using this and (3.40) in (3.32), we have

$$
D \phi_{\mu_{k}}\left(x_{k} ; d_{k}\right) \leq-\left\|Z_{k}^{T} g_{k}+\zeta_{k} w_{k}\right\|\left\|p_{\mathrm{Z}}\right\| \cos \theta_{k}-\rho_{k}\left\|c_{k}\right\|_{1} .
$$

The directional derivative is thus nonpositive. Furthermore, since $w_{k}=0$ whenever $c_{k}=0$ (regardless of whether $w_{k}$ is obtained by finite differences or through Broyden's method), it is easy to show that this directional derivative can be zero only at a stationary point of problem $(1.1)-(1.2)$.

\subsection{The Algorithm}

We can now give a complete description of the algorithm that incorporates all the ideas discussed so far and that specifies the only remaining question, namely, when to apply finite 
differences and when to use Broyden's method to approximate the cross term. The idea is to consider the relative sizes of $p_{\mathrm{Y}}$ and $p_{\mathrm{Z}}$. Update Criterion I generates the three regions $R_{1}, R_{2}$, and $R_{3}$ illustrated in Figure 2. The algorithm starts by computing $w_{k}$ through Broyden's method and by calculating $p_{\mathrm{Y}}$ and $p_{\mathrm{Z}}$. If the search direction is in $R_{1}$ or $R_{3}$, we proceed. Otherwise we recompute $w_{k}$ by finite differences, use this value to recompute $p_{\mathrm{Z}}$, and proceed. The reason for applying finite differences in this fashion is that in the middle region $R_{2}$ Broyden's method is not good enough, nor is the convergence sufficiently tangential, to give a superlinear step. Therefore we must resort to finite differences to obtain a good estimate of $w_{k}$. The motivation behind this strategy will become clearer when we study the rate of convergence of the algorithm in $\S 6$.

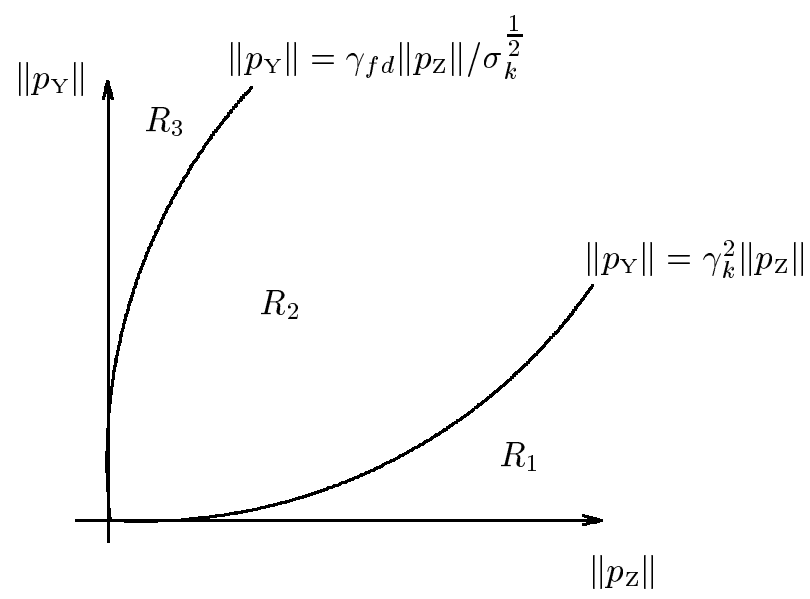

Figure 2

Three regions generated by Update Criterion I

Note from Updating Criterion I that the BFGS update of $B_{k}$ is skipped if the search direction is in $R_{3}$. A precise description of the algorithm follows.

\section{Algorithm II}

1. Choose constants $\eta \in(0,1 / 2), \rho>0$ and $\tau$, $\tau^{\prime}$ with $0<\tau<\tau^{\prime}<1$, and positive constants $\Gamma$ and $\gamma_{\mathrm{fd}}$ for Conditions (3.13) and (3.19), respectively. For Conditions (3.14) and (3.18), select a summable sequence of positive numbers $\left\{\gamma_{k}\right\}$. Set $k:=1$, and choose a starting point $x_{1}$, an initial value $\mu_{1}$ for the penalty parameter, an $(n-m) \times(n-m)$ symmetric and positive definite starting matrix $B_{1}$ and an $(n-m) \times n$ starting matrix $S_{1}$.

2. Evaluate $f_{k}, g_{k}, c_{k}$, and $A_{k}$, and compute $Y_{k}$ and $Z_{k}$.

3. Set findiff $=$ false and compute $p_{\mathrm{Y}}$ by solving the system

$$
\left(A_{k}^{T} Y_{k}\right) p_{\mathrm{Y}}=-c_{k} . \quad \text { (range space step) }
$$

4. Calculate $w_{k}$ using Broyden's method, from Equations (3.12) and (3.13). 
5. Choose the damping parameter $\zeta_{k}$ from Equations (3.38) and (3.39), and compute $p_{\mathrm{Z}}$ from

$$
B_{k} p_{\mathrm{Z}}=-\left[Z_{k}^{T} g_{k}+\zeta_{k} w_{k}\right] . \quad \text { (null space step) }
$$

6. If (3.19) is satisfied and (3.18) is not satisfied, set findiff $=$ true and recompute $w_{k}$ from Equation (3.1). (In practice we replace $\sigma_{k}$ by $\left\|Z_{k}^{T} g_{k}\right\|+\left\|c_{k}\right\|$ in (3.19).)

7. If findiff $=$ true, use this new value of $w_{k}$ to choose the damping parameter $\zeta_{k}$ from Equations (3.38) and (3.39), and recompute $p_{\mathrm{Z}}$ from Equation (3.43).

8. Define the search direction by

$$
d_{k}=Y_{k} p_{\mathrm{Y}}+Z_{k} p_{\mathrm{Z}}
$$

and set $\alpha_{k}=1$.

9. Test the line search condition

$$
\phi_{\mu_{k}}\left(x_{k}+\alpha_{k} d_{k}\right) \leq \phi_{\mu_{k}}\left(x_{k}\right)+\eta \alpha_{k} D \phi_{\mu_{k}}\left(x_{k} ; d_{k}\right) .
$$

10. If (3.45) is not satisfied, choose a new $\alpha_{k} \in\left[\tau \alpha_{k}, \tau^{\prime} \alpha_{k}\right]$ and go to 9 ; otherwise set

$$
x_{k+1}=x_{k}+\alpha_{k} d_{k} \text {. }
$$

11. Evaluate $f_{k+1}, g_{k+1}, c_{k+1}, A_{k+1}$, and compute $Y_{k+1}$ and $Z_{k+1}$.

12. Compute the Lagrange multiplier estimate

$$
\lambda_{k+1}=-\left[Y_{k+1}^{T} A_{k+1}\right]^{-1} Y_{k+1}^{T} g_{k+1},
$$

and update $\mu_{k}$ so as to satisfy (3.40).

13. Update $S_{k+1}$ using Equations (3.9) to (3.11). If findiff $=$ false, calculate $\bar{w}_{k}$ by Broyden's method through Equations (3.12) and (3.14); otherwise calculate $\bar{w}_{k}$ by (3.2).

14. If $\left(s_{k}^{T} y_{k} \leq 0\right)$ or if (findif $f=$ true and (3.19) is not satisfied) or if (findiff $=$ false and (3.18) is not satisfied), set $B_{k+1}=B_{k}$. Else, compute

$$
\begin{gathered}
s_{k}=\alpha_{k} p_{\mathrm{Z}}, \\
y_{k}=Z_{k}^{T}\left[\nabla L\left(x_{k+1}, \lambda_{k+1}\right)-\nabla L\left(x_{k}, \lambda_{k+1}\right)\right]-\bar{w}_{k},
\end{gathered}
$$

and compute $B_{k+1}$ by the BFGS formula (1.24).

15. Set $k:=k+1$, and go to 3 .

We mentioned in $\S 3.1$ that, when using finite differences, there are various ways of defining $w_{k}$ and $\bar{w}_{k}$, but for concreteness we now assume in steps 6 and 13 that they are computed by (3.1) and (3.2), respectively. We should also point out that the curves in Figure 2 may intersect, creating a fourth region, and in practice we should stipulate a new set of conditions in this region. We discuss these conditions in another paper that considers the implementation of the algorithm (Biegler, Nocedal, and Schmid (1993)).

In the next sections we present several convergence results for Algorithm II. The analysis, which does not assume that the BFGS matrices $B_{k}$ or the Broyden matrices $S_{k}$ are bounded, is based on the results of Byrd and Nocedal (1991), who have studied the convergence of the Coleman-Conn updating algorithm. We also make use of some results of Xie (1991), who has 
analyzed the algorithm proposed by Nocedal and Overton (1985) using nonorthogonal bases $Y$ and $Z$. The main difference between this paper and that of Xie stems from our use of the correction terms $w_{k}$ and $\bar{w}_{k}$, which are not employed in his method.

\section{Semi-Local Behavior of the Algorithm}

We first show that the merit function $\phi$ decreases significantly at the good iterates $J$ and that this gives the algorithm a weak convergence property. To establish the results of this section, we make the following assumptions.

Assumptions 4.1 The sequence $\left\{x_{k}\right\}$ generated by Algorithm II is contained in a convex set $D$ with the following properties:

(I) The functions $f: \mathbf{R}^{n} \rightarrow \mathbf{R}$ and $c: \mathbf{R}^{n} \rightarrow \mathbf{R}^{m}$ and their first and second derivatives are uniformly bounded in norm over $D$.

(II) The matrix $A(x)$ has full column rank for all $x \in D$, and there exist constants $\gamma_{0}$ and $\beta_{0}$ such that

$$
\left\|Y(x)\left[A(x)^{T} Y(x)\right]^{-1}\right\| \leq \gamma_{0}, \quad\|Z(x)\| \leq \beta_{0},
$$

for all $x \in D$.

(III) For all $k \geq 1$ for which $B_{k}$ is updated, (3.21) and (3.22) hold.

(IV) The correction term $w_{k}$ is chosen so that there is a constant $\kappa>0$ such that for all $k$,

$$
\left\|w_{k}\right\| \leq \kappa\left\|c_{k}\right\|^{1 / 2} .
$$

Note that Condition (I) is rather strong, since it would often be satisfied only if $D$ is bounded, and it is far from certain that the iterates will remain in a bounded set. Nevertheless, the convergence result of this section can be combined with the local analysis of $\S 5$ to give a satisfactory semi-global result. Condition (II) requires that the basis matrices $Y$ and $Z$ be chosen carefully, and is important to obtain good behavior in practice. Note that (4.1) and (3.42) imply that

$$
\left\|Y_{k} p_{\mathrm{Y}}\right\| \leq \gamma_{0}\left\|c_{k}\right\|
$$

Condition (III) is justified by Lemma 3.1. Condition (III) and Theorem 3.1 ensure that at least half of the iterates at which BFGS updating takes place are good iterates.

We have left some freedom in the choice of $w_{k}$ since (4.2) suffices for the analysis of this section. Relation (4.2) holds for the finite-difference approach, since (3.1) implies that $w_{k}=O\left(Y_{k} p_{\mathrm{Y}}\right)$ and since (I) ensures that $\left\{\left\|c_{k}\right\|\right\}$ is uniformly bounded (see (5.21)). Furthermore, the safeguard (3.13) and (4.3) immediately imply that (4.2) is satisfied when the Broyden approximation is used.

The following result concerns the good iterates $J$, as given in Definition 3.1.

Lemma 4.1 If Assumptions 4.1 hold and if $\mu_{j}=\mu$ is constant for all sufficiently large $j$, then there is a positive constant $\gamma_{\mu}$ such that for all large $j \in J$,

$$
\phi_{\mu}\left(x_{j}\right)-\phi_{\mu}\left(x_{j+1}\right) \geq \gamma_{\mu}\left[\left\|Z_{j}^{T} g_{j}\right\|^{2}+\left\|c_{j}\right\|_{1}\right] .
$$


Proof. Using (3.37), we have for all $j \in J$

$$
D \phi_{\mu_{j}}\left(x_{j} ; d_{j}\right) \leq-b_{2}\left[\left\|Z_{j}^{T} g_{j}\right\|^{2}+\left\|c_{j}\right\|_{1}\right]
$$

where $b_{2}=\min \left(\beta_{1} / \beta_{3}, \rho\right)$. Note that the line search enforces the Armijo condition (3.45),

$$
\phi_{\mu_{j}}\left(x_{j}\right)-\phi_{\mu_{j}}\left(x_{j+1}\right) \geq-\eta \alpha_{j} D \phi_{\mu_{j}}\left(x_{j} ; d_{j}\right) .
$$

It is then clear from (4.5) that (4.4) holds, provided the $\alpha_{j}, j \in J$, can be bounded from below. Suppose that $\alpha_{j}<1$, which means that (4.6) failed for a steplength $\tilde{\alpha}$ :

$$
\phi_{\mu_{j}}\left(x_{j}+\tilde{\alpha} d_{j}\right)-\phi_{\mu_{j}}\left(x_{j}\right)>\eta \tilde{\alpha} D \phi_{\mu_{j}}\left(x_{j} ; d_{j}\right),
$$

where

$$
\tau \tilde{\alpha} \leq \alpha_{j}
$$

(see Step 10 of Algorithm II). On the other hand, expanding to second order, we have

$$
\phi_{\mu_{j}}\left(x_{j}+\tilde{\alpha} d_{j}\right)-\phi_{\mu_{j}}\left(x_{j}\right) \leq \tilde{\alpha} D \phi_{\mu_{j}}\left(x_{j} ; d_{j}\right)+\tilde{\alpha}^{2} b_{1}\left\|d_{j}\right\|^{2},
$$

where $b_{1}$ depends on $\mu_{j}$. Combining (4.7) and (4.9), we have

$$
(\eta-1) \tilde{\alpha} D \phi_{\mu_{j}}\left(x_{j} ; d_{j}\right)<\tilde{\alpha}^{2} b_{1}\left\|d_{j}\right\|^{2} .
$$

Next we show that, for $j \in J$,

$$
\left\|d_{j}\right\|^{2} \leq b_{3}\left[\left\|Z_{j}^{T} g_{j}\right\|^{2}+\left\|c_{j}\right\|_{1}\right]
$$

for some constant $b_{3}$. To do this, we make repeated use of the following elementary result:

$$
a, b \geq 0 \Rightarrow a^{2}+2 a b+b^{2} \leq 3 a^{2}+3 b^{2} .
$$

Using (3.44), (4.12), (4.1), and (4.3), we have

$$
\begin{aligned}
\left\|d_{j}\right\|^{2} & \leq\left\|Z_{j} p_{\mathrm{Z}}^{(j)}\right\|^{2}+2\left\|Z_{j} p_{\mathrm{Z}}^{(j)}\right\|\left\|Y_{j} p_{\mathrm{Y}}^{(j)}\right\|+\left\|Y_{j} p_{\mathrm{Y}}^{(j)}\right\|^{2} \\
& \leq 3\left[\left\|Z_{j} p_{\mathrm{Z}}^{(j)}\right\|^{2}+\left\|Y_{j} p_{\mathrm{Y}}^{(j)}\right\|^{2}\right] \\
& \leq 3\left[\beta_{0}^{2}\left\|p_{\mathrm{Z}}^{(j)}\right\|^{2}+\gamma_{0}^{2}\left\|c_{j}\right\|^{2}\right] .
\end{aligned}
$$

Also by (3.34), (4.12), and (4.2) and noting that $\|\cdot\| \leq\|\cdot\|_{1}$, we have that for $j \in J$

$$
\begin{aligned}
\left\|p_{\mathrm{Z}}^{(j)}\right\|^{2} & \leq \frac{1}{\beta_{2}^{2}}\left[\left\|Z_{j}^{T} g_{j}\right\|^{2}+2 \zeta_{j}\left\|Z_{j}^{T} g_{j}\right\|\left\|w_{j}\right\|+\zeta_{j}^{2}\left\|w_{j}\right\|^{2}\right] \\
& \leq \frac{3}{\beta_{2}^{2}}\left[\left\|Z_{j}^{T} g_{j}\right\|^{2}+\zeta_{j}^{2}\left\|w_{j}\right\|^{2}\right] \\
& \leq \frac{3}{\beta_{2}^{2}}\left[\left\|Z_{j}^{T} g_{j}\right\|^{2}+\kappa^{2}\left\|c_{j}\right\|_{1}\right]
\end{aligned}
$$

since $\zeta_{j} \leq 1$. Since $\left\|c_{j}\right\|_{1}$ is uniformly bounded on $D$, we see from this relation and (4.13) that (4.11) holds, where

$$
b_{3}=\max \left\{9 \beta_{0}^{2} / \beta_{2}^{2}, 3\left(3 \kappa^{2} \beta_{0}^{2} / \beta_{2}^{2}+\gamma_{0}^{2} \sup _{x \in D}\|c(x)\|\right)\right\} .
$$


Combining (4.10), (4.5), and (4.11), and recalling that $\eta<1$, we obtain

$$
\tilde{\alpha}>\frac{(1-\eta) b_{2}}{b_{1} b_{3}} .
$$

This relation and (4.8) imply that the steplengths $\alpha_{j}$ are bounded away from zero for all $j \in J$. Since by assumption $\mu_{j}=\mu$ for all large $j$, we conclude that (4.4) holds with $\gamma_{\mu}=\eta b_{2} \min \{1,(1-$ $\left.\eta) \tau b_{2} /\left(b_{1} b_{3}\right)\right\}$.

It is now easy to show that the penalty parameter settles down and that the set of iterates is not bounded away from stationary points of the problem.

Theorem 4.2 If Assumptions 4.1 hold, then the weights $\left\{\mu_{k}\right\}$ are constant for all sufficiently large $k$ and

$$
\liminf _{k \rightarrow \infty}\left(\left\|Z_{k}^{T} g_{k}\right\|+\left\|c_{k}\right\|\right)=0
$$

Proof. First note that by Assumptions 4.1 (I)-(II) and (3.47) that $\left\{\left\|\lambda_{k}\right\|\right\}$ is bounded. Therefore, since the procedure (3.40) increases $\mu_{k}$ by at least $\rho$ whenever it changes the penalty parameter, it follows that there are an index $k_{0}$ and a value $\mu$ such that for all $k>k_{0}, \mu_{k}=\mu \geq\left\|\lambda_{k}\right\|+2 \rho$.

If BFGS updating is performed an infinite number of times, by Assumptions 4.1-(III) and Theorem 3.1 there is an infinite set $J$ of good iterates, and by Lemma 4.1 and the fact that the Armijo condition (3.45) forces $\phi_{\mu}\left(x_{k}\right)$ to decrease at each iterate, we have that for $k>k_{0}$,

$$
\begin{aligned}
\phi_{\mu}\left(x_{k_{0}}\right)-\phi_{\mu}\left(x_{k+1}\right) & =\sum_{j=k_{0}}^{k}\left(\phi_{\mu}\left(x_{j}\right)-\phi_{\mu}\left(x_{j+1}\right)\right) \\
& \geq \sum_{j \in J \cap\left[k_{0}, k\right]}\left(\phi_{\mu}\left(x_{j}\right)-\phi_{\mu}\left(x_{j+1}\right)\right) \\
& \geq \gamma_{\mu} \sum_{j \in J \cap\left[k_{0}, k\right]}\left[\left\|Z_{j}^{T} g_{j}\right\|^{2}+\left\|c_{j}\right\|_{1}\right] .
\end{aligned}
$$

By Assumption 4.1(I) $\phi_{\mu}(x)$ is bounded below for all $x \in D$, so the last sum is finite, and thus the term inside the square brackets converges to zero. Therefore

$$
\lim _{\substack{j \in J \\ j \rightarrow \infty}}\left(\left\|Z_{j}^{T} g_{j}\right\|+\left\|c_{j}\right\|_{1}\right)=0 .
$$

If BFGS updating is performed a finite number of times, then, as discussed after Definition 3.1, all iterates are good iterates, and in this case we obtain the stronger result

$$
\lim _{k \rightarrow \infty}\left(\left\|Z_{k}^{T} g_{k}\right\|+\left\|c_{k}\right\|_{1}\right)=0 .
$$

\section{Local Convergence}

In this section we show that if $x_{*}$ is a local minimizer that satisfies the second-order optimality conditions, and if the penalty parameter $\mu_{k}$ is chosen large enough, then $x_{*}$ is a point of attraction 
for the sequence of iterates $\left\{x_{k}\right\}$ generated by Algorithm II. To prove this result, we will make the following assumptions. In what follows, $G$ denotes the reduced Hessian of the Lagrangian function, namely,

$$
G_{k}=Z_{k}^{T} \nabla_{x x}^{2} L\left(x_{k}, \lambda_{k}\right) Z_{k}
$$

Assumptions 5.1 The point $x_{*}$ is a local minimizer for problem (1.1)-(1.2), at which the following conditions hold.

(1) The functions $f: \mathbf{R}^{n} \rightarrow \mathbf{R}$ and $c: \mathbf{R}^{n} \rightarrow \mathbf{R}^{m}$ are twice continuously differentiable in a neighborhood of $x_{*}$, and their Hessians are Lipschitz continuous in a neighborhood of $x_{*}$.

(2) The matrix $A\left(x_{*}\right)$ has full column rank. This implies that there exists a vector $\lambda_{*} \in \mathbf{R}^{m}$ such that

$$
\nabla L\left(x_{*}, \lambda_{*}\right)=g\left(x_{*}\right)+A\left(x_{*}\right) \lambda_{*}=0 .
$$

(3) For all $q \in \mathbf{R}^{n-m}, q \neq 0$, we have $q^{T} G_{*} q>0$.

(4) There exist constants $\gamma_{0}, \beta_{0}$, and $\gamma_{c}$ such that, for all $x$ in a neighborhood of $x_{*}$,

$$
\left\|Y(x)\left[A(x)^{T} Y(x)\right]^{-1}\right\| \leq \gamma_{0}, \quad\|Z(x)\| \leq \beta_{0},
$$

and

$$
\left\|[Y(x) Z(x)]^{-1}\right\| \leq \gamma_{c} .
$$

(5) $Z(x)$ and $\lambda(x)$ are Lipschitz continuous in a neighborhood of $x_{*}$. That is, there exist constants $\gamma_{\mathrm{Z}}$ and $\gamma_{\lambda}$ such that

$$
\begin{aligned}
\|\lambda(x)-\lambda(z)\| & \leq \gamma_{\lambda}\|x-z\|, \\
\|Z(x)-Z(z)\| & \leq \gamma_{\mathrm{Z}}\|x-z\|,
\end{aligned}
$$

for all $x, z$ near $x_{*}$.

Note that (1), (3), and (5) imply that for all $(x, \lambda)$ sufficiently near $\left(x_{*}, \lambda_{*}\right)$, and for all $q \in \mathbf{R}^{n-m}$,

$$
m\|q\|^{2} \leq q^{T} G(x, \lambda) q \leq M\|q\|^{2},
$$

for some positive constants $m, M$. We also note that Assumptions 5.1 ensure that the conditions (3.21)-(3.22) required by Theorem 3.1 hold whenever BFGS updating takes place in a neighborhood of $x_{*}$, as shown in Lemma 3.1. Therefore Theorem 3.1 can be applied in the convergence analysis.

The following two lemmas are proved by Xie (1991) for very general choices of $Y$ and $Z$. His result generalizes Lemmas 4.1 and 4.2 of Byrd and Nocedal (1991); see also Powell (1978).

Lemma 5.1 If Assumptions 5.1 hold, then for all $x$ sufficiently near $x_{*}$

$$
\gamma_{1}\left\|x-x_{*}\right\| \leq\|c(x)\|+\left\|Z(x)^{T} g(x)\right\| \leq \gamma_{2}\left\|x-x_{*}\right\|,
$$

for some positive constants $\gamma_{1}, \gamma_{2}$.

This result states that, near $x_{*}$, the quantities $c(x)$ and $Z(x)^{T} g(x)$ may be regarded as a measure of the error at $x$. The next lemma states that, for a large enough weight, the merit function may also be regarded as a measure of the error. 
Lemma 5.2 Suppose that Assumptions 5.1 hold at $x_{*}$. Then for any $\mu>\left\|\lambda_{*}\right\|_{\infty}$ there exist constants $\gamma_{3}>0$ and $\gamma_{4}>0$, such that for all $x$ sufficiently near $x_{*}$

$$
\gamma_{3}\left\|x-x_{*}\right\|^{2} \leq \phi_{\mu}(x)-\phi_{\mu}\left(x_{*}\right) \leq \gamma_{4}\left[\left\|Z(x)^{T} g(x)\right\|^{2}+\|c(x)\|_{1}\right] .
$$

Note that the left inequality in (5.8) implies that, for a sufficiently large value of the penalty parameter, the merit function will have a strong local minimizer at $x_{*}$. We will now use the descent property of Algorithm II to show convergence of the algorithm. However, because of the nonconvexity of the problem, the line search could generate a step that decreases the merit function but that takes us away from the neighborhood of $x_{*}$. To rule this out, we make the following assumption.

Assumption 5.2 The line search has the property that, for all large $k, \phi_{\mu}\left((1-\theta) x_{k}+\theta x_{k+1}\right) \leq$ $\phi_{\mu}\left(x_{k}\right)$ for all $\theta \in[0,1]$. In other words, $x_{k+1}$ is in the connected component of the level set $\left\{x: \phi_{\mu}(x) \leq \phi_{\mu}\left(x_{k}\right)\right\}$ that contains $x_{k}$.

There is no practical line search algorithm that can guarantee this condition, but it is likely to hold close to $x_{*}$. Assumption 5.2 is made by Byrd, Nocedal, and Yuan (1987) when analyzing the convergence of variable metric methods for unconstrained problems, as well as by Byrd and Nocedal (1991) in the analysis of Coleman-Conn updates for equality constrained optimization.

Lemma 5.3 Suppose that the iterates generated by Algorithm II (with a line search satisfying Assumptions 5.2) are contained in a convex region D satisfying Assumptions 4.1. If an iterate $x_{k_{0}}$ is sufficiently close to a solution point $x_{*}$ that satisfies Assumptions 5.1, and if the weight $\mu_{k_{0}}$ is large enough, then the sequence of iterates converges to $x_{*}$.

Proof. By Assumptions 4.1 (I)-(II) and (3.47) we know that $\left\{\left\|\lambda_{k}\right\|\right\}$ is bounded. Therefore the procedure (3.40) ensures that the weights $\mu_{k}$ are constant, say $\mu_{k}=\mu$ for all large $k$. Moreover, if an iterate gets sufficiently close to $x_{*}$, we know by (3.40) and by the continuity of $\lambda$ that $\mu>\left\|\lambda_{*}\right\|$. For such value of $\mu$, Lemma 5.2 implies that the merit function has a strict local minimizer at $x_{*}$. Now suppose that once the penalty parameter has settled, and for a given $\epsilon>0$, there is an iterate $x_{k_{0}}$ such that

$$
\left\|x_{k_{0}}-x_{*}\right\| \leq \frac{\gamma_{3}}{\gamma_{2} \gamma_{4} \hat{\gamma}_{0}} \epsilon^{2}
$$

where $\hat{\gamma}_{0}$ is such that $\|\cdot\|_{1} \leq \hat{\gamma}_{0}\|\cdot\|$. Assumption 5.2 shows that for any $k \geq k_{0}, x_{k}$ is in the connected component of the level set of $x_{k_{0}}$ that contains $x_{k_{0}}$, and we can assume that $\epsilon$ is small enough that Lemmas 5.1 and 5.2 hold in this level set. Thus since $\phi_{\mu}\left(x_{k}\right) \leq \phi_{\mu}\left(x_{k_{0}}\right)$ for $k \geq k_{0}$, and since we can assume that $\left\|Z_{k_{0}}^{T} g_{k_{0}}\right\| \leq 1$, we have from Lemmas 5.1 and 5.2 , for any $k \geq k_{0}$

$$
\begin{aligned}
\left\|x_{k}-x_{*}\right\| & \leq \gamma_{3}^{-\frac{1}{2}}\left(\phi_{\mu}\left(x_{k}\right)-\phi_{\mu}\left(x_{*}\right)\right)^{\frac{1}{2}} \\
& \leq \gamma_{3}^{-\frac{1}{2}}\left(\phi_{\mu}\left(x_{k_{0}}\right)-\phi_{\mu}\left(x_{*}\right)\right)^{\frac{1}{2}} \\
& \leq\left(\frac{\gamma_{4}}{\gamma_{3}}\right)^{\frac{1}{2}}\left[\left\|Z_{k_{0}}^{T} g_{k_{0}}\right\|^{2}+\left\|c_{k_{0}}\right\|_{1}\right]^{\frac{1}{2}} \\
& \leq\left(\frac{\gamma_{4}}{\gamma_{3}}\right)^{\frac{1}{2}}\left[\left\|Z_{k_{0}}^{T} g_{k_{0}}\right\|^{2}+\hat{\gamma}_{0}\left\|c_{k_{0}}\right\|\right]^{\frac{1}{2}}
\end{aligned}
$$




$$
\begin{aligned}
& \leq\left(\frac{\gamma_{2} \gamma_{4} \hat{\gamma}_{0}}{\gamma_{3}}\left\|x_{k_{0}}-x_{*}\right\|\right)^{\frac{1}{2}} \\
& \leq \epsilon
\end{aligned}
$$

This implies that the whole sequence of iterates remains in a neighborhood of radius $\epsilon$ of $x_{*}$. If $\epsilon$ is small enough, we conclude by (5.8), by the monotonicity of $\left\{\phi_{\mu}\left(x_{k}\right)\right\}$, and by Theorem 4.2 that the iterates converge to $x_{*}$.

The assumptions of this lemma, which is modeled after a result in Xie (1991), are restrictive - especially the assumption on the penalty parameter. One can relax these assumptions and obtain a stronger result, such as Theorem 4.3 in Byrd and Nocedal (1991), but the proof would be more complex and is not particularly relevant to Algorithm II since it is based only on the properties of the merit function. Therefore, instead of further analyzing the local convergence properties of the new algorithm, we will study its rate of convergence.

\subsection{R-Linear Convergence}

For the rest of the paper we assume that the line search strategy satisfies Assumption 5.2. We also assume that the iterates generated by Algorithm II converge to a point $x_{*}$ at which Assumptions 5.1 hold, which implies that for all large $k, \mu_{k}=\mu>\left\|\lambda_{*}\right\|$. The analysis that follows depends on how often BFGS updating is applied. To make this concept precise, we define $U$ to be the set of iterates at which BFGS updating takes place,

$$
U=\left\{k: B_{k+1}=B F G S\left(B_{k}, s_{k}, y_{k}\right)\right\},
$$

and let

$$
U_{k}=U \cap\{1,2, \ldots, k\} .
$$

The number of elements in $U_{k}$ will be denoted by $\left|U_{k}\right|$.

Theorem 5.4 Suppose that the iterates $\left\{x_{k}\right\}$ generated by Algorithm II converge to a point $x_{*}$ that satisfies Assumptions 5.1. Then for any $k \in U$ and any $j \geq k$

$$
\left\|x_{j}-x_{*}\right\| \leq C r^{\left|U_{k}\right|}
$$

for some constants $C>0$ and $0 \leq r<1$.

Proof. Using (4.4) and (5.8), we have for $i \in J$,

$$
\phi_{\mu}\left(x_{i}\right)-\phi_{\mu}\left(x_{i+1}\right) \geq \frac{\gamma_{\mu}}{\gamma_{4}}\left[\phi_{\mu}\left(x_{i}\right)-\phi_{\mu}\left(x_{*}\right)\right] .
$$

Let us define $r=\left(1-\gamma_{\mu} / \gamma_{4}\right)^{\frac{1}{4}}$. Then for $i \in J$

$$
\phi_{\mu}\left(x_{i+1}\right)-\phi_{\mu}\left(x_{*}\right) \leq r^{4}\left[\phi_{\mu}\left(x_{i}\right)-\phi_{\mu}\left(x_{*}\right)\right] .
$$

We know that the merit function decreases at each step, and by (5.8) we have, for $j \geq k$ and $k \in U$,

$$
\begin{aligned}
\left\|x_{j}-x_{*}\right\| & \leq \gamma_{3}^{-\frac{1}{2}}\left(\phi_{\mu}\left(x_{j}\right)-\phi_{\mu}\left(x_{*}\right)\right)^{\frac{1}{2}} \\
& \leq \gamma_{3}^{-\frac{1}{2}}\left(\phi_{\mu}\left(x_{k}\right)-\phi_{\mu}\left(x_{*}\right)\right)^{\frac{1}{2}} .
\end{aligned}
$$


We continue in this fashion, bounding the right-hand side by terms involving earlier iterates, but using now (5.13) for all good iterates. Since by Theorem 3.1 at least half of the iterates at which updating takes place are good iterates (i.e., $\left|J_{k}\right| \geq \frac{1}{2}\left|U_{k}\right|$ ), we have

$$
\begin{aligned}
\left\|x_{j}-x_{*}\right\| & \leq \gamma_{3}^{-\frac{1}{2}}\left[r^{4\left|J_{k}\right|}\left(\phi_{\mu}\left(x_{1}\right)-\phi_{\mu}\left(x_{*}\right)\right)\right]^{\frac{1}{2}} \\
& \leq \gamma_{3}^{-\frac{1}{2}}\left[r^{2\left|U_{k}\right|}\left(\phi_{\mu}\left(x_{1}\right)-\phi_{\mu}\left(x_{*}\right)\right)\right]^{\frac{1}{2}} \\
& \leq\left[\gamma_{3}^{-\frac{1}{2}}\left(\phi_{\mu}\left(x_{1}\right)-\phi_{\mu}\left(x_{*}\right)\right)^{\frac{1}{2}}\right] r^{\left|U_{k}\right|} \\
& \equiv C r^{\left|U_{k}\right|} .
\end{aligned}
$$

This result implies that if $\left\{\left|U_{k}\right| / k\right\}$ is bounded away from zero, then Algorithm II is R-linearly convergent. However, BFGS updating could take place only a finite number of times, in which case this ratio would converge to zero. It is also possible for BFGS updating to take place an infinite number of times, but every time less often, in such a way that $\left|U_{k}\right| / k \rightarrow 0$. We therefore need to examine the iteration more closely.

We make use of the matrix function $\psi$ defined by

$$
\psi(B)=\operatorname{tr}(B)-\ln (\operatorname{det}(B)),
$$

where $t r$ denotes the trace, and det the determinant. It can be shown that

$$
\ln \operatorname{cond}(B)<\psi(B)
$$

for any positive definite matrix $B$ (Byrd and Nocedal (1989)). We also make use of the weighted quantities

$$
\begin{gathered}
\tilde{y}_{k}=G_{*}^{-1 / 2} y_{k}, \quad \tilde{s}_{k}=G_{*}^{1 / 2} s_{k}, \\
\tilde{B}_{k}=G_{*}^{-1 / 2} B_{k} G_{*}^{-1 / 2}, \\
\cos \tilde{\theta}_{k}=\frac{\tilde{s}_{k}^{T} \tilde{B}_{k} \tilde{s}_{k}}{\left\|\tilde{B}_{k} \tilde{s}_{k}\right\|\left\|\tilde{s}_{k}\right\|},
\end{gathered}
$$

and

$$
\tilde{q}_{k}=\frac{\tilde{s}_{k}^{T} \tilde{B}_{k} \tilde{s}_{k}}{\tilde{s}_{k}^{T} \tilde{s}_{k}}
$$

One can show (see Eq. (3.22) of Byrd and Nocedal (1989)) that if $B_{k}$ is updated by the BFGS formula, then

$$
\begin{aligned}
\psi\left(\tilde{B}_{k+1}\right)= & \psi\left(\tilde{B}_{k}\right)+\frac{\left\|\tilde{y}_{k}\right\|^{2}}{\tilde{y}_{k}^{T} \tilde{s}_{k}}-1-\ln \frac{\tilde{y}_{k}^{T} \tilde{s}_{k}}{\tilde{s}_{k}^{T} \tilde{s}_{k}}+\ln \cos ^{2} \tilde{\theta}_{k} \\
& +\left[1-\frac{\tilde{q}_{k}}{\cos ^{2} \tilde{\theta}_{k}}+\ln \frac{\tilde{q}_{k}}{\cos ^{2} \tilde{\theta}_{k}}\right] .
\end{aligned}
$$

This expression characterizes the behavior of the BFGS matrices $B_{k}$ and will be crucial to the analysis of this section. Before we can make use of this relation, however, we need to consider the accuracy of the correction terms. We begin by showing that when finite differences are used to estimate $w_{k}$ and $\bar{w}_{k}$, these are accurate to second order. 
Lemma 5.5 If at the iterate $x_{k}$, the corrections $w_{k}$ and $\bar{w}_{k}$ are computed by the finite-difference formulae (3.1)-(3.2), and if $x_{k}$ is sufficiently close to a solution point $x_{*}$ that satisfies Assumptions 5.1, then

$$
\begin{gathered}
w_{k}=O\left(\left\|p_{\mathrm{Y}}\right\|\right), \\
\left\|w_{k}-Z_{*}^{T} W_{*} Y_{k} p_{\mathrm{Y}}\right\|=O\left(\sigma_{k}\left\|p_{\mathrm{Y}}\right\|\right)
\end{gathered}
$$

and

$$
\left\|\bar{w}_{k}-\alpha_{k} Z_{*}^{T} W_{*} Y_{k} p_{\mathrm{Y}}\right\|=O\left(\sigma_{k}\left\|p_{\mathrm{Y}}\right\|\right) .
$$

Proof. Recalling that $\nabla L(x, \lambda)=g(x)+A(x) \lambda$, we have from (3.1) that

$$
\begin{aligned}
w_{k} & =Z_{k}^{T}\left[\nabla L\left(x_{k}+Y_{k} p_{\mathrm{Y}}, \lambda_{k}\right)-\nabla L\left(x_{k}, \lambda_{k}\right)\right] \\
& =Z_{k}^{T}\left[\nabla L\left(x_{k}+Y_{k} p_{\mathrm{Y}}, \lambda_{*}\right)-\nabla L\left(x_{k}, \lambda_{*}\right)\right]+Z_{k}^{T}\left[\left(A\left(x_{k}+Y_{k} p_{\mathrm{Y}}\right)-A_{k}\right)\left(\lambda_{k}-\lambda_{*}\right)\right] \\
& =Z_{k}^{T}\left[\int_{0}^{1} \nabla_{x x}^{2} L\left(x_{k}+\tau Y_{k} p_{\mathrm{Y}}, \lambda_{*}\right) d \tau\right] Y_{k} p_{\mathrm{Y}}+Z_{k}^{T}\left[\left(A\left(x_{k}+Y_{k} p_{\mathrm{Y}}\right)-A_{k}\right)\left(\lambda_{k}-\lambda_{*}\right)\right] \\
& \equiv Z_{k}^{T} \bar{W}_{k} Y_{k} p_{\mathrm{Y}}+Z_{k}^{T}\left[\left(A\left(x_{k}+Y_{k} p_{\mathrm{Y}}\right)-A_{k}\right)\left(\lambda_{k}-\lambda_{*}\right)\right] .
\end{aligned}
$$

Let us assume that $x_{k}$ is in the neighborhood of $x_{*}$ where (5.2)-(5.5) hold. Then $\left\|\lambda_{k}-\lambda_{*}\right\|=$ $O\left(\left\|e_{k}\right\|\right)=O\left(\sigma_{k}\right)$, where $\sigma_{k}$ is defined by (1.34). Therefore the last term in (5.24) is $O\left(\left\|p_{\mathrm{Y}}\right\| \sigma_{k}\right)$, which proves (5.21). Also, a simple computation shows that

$$
\left[Z_{k}^{T} \bar{W}_{k}-Z_{*}^{T} W_{*}\right] Y_{k} p_{\mathrm{Y}}=O\left(\sigma_{k}\left\|p_{\mathrm{Y}}\right\|\right)
$$

Using these facts in (5.24) yields the desired result (5.22). To prove (5.23), we note only that $\alpha_{k} \leq 1$ and reason in the same manner.

Next we show that the condition number of the matrices $B_{k}$ is bounded and that, in the limit, at the iterates $U$ at which BFGS updating takes place, the matrices $B_{k}$ are accurate approximations of the reduced Hessian of the Lagrangian.

Theorem 5.6 Suppose that the iterates $\left\{x_{k}\right\}$ generated by Algorithm II converge to a solution point $x_{*}$ that satisfies Assumptions 5.1. Then $\left\{\left\|B_{k}\right\|\right\}$ and $\left\{\left\|B_{k}^{-1}\right\|\right\}$ are bounded, and for all $k \in U$

$$
\left\|\left(B_{k}-Z_{*}^{T} W_{*} Z_{*}\right) p_{Z}\right\|=o\left(\left\|d_{k}\right\|\right) .
$$

Proof. We will only consider iterates $k$ for which BFGS updating of $B_{k}$ takes place. We have from (3.49), (3.46), (3.44), (3.16), and (3.48)

$$
\begin{aligned}
y_{k} & =Z_{k}^{T}\left[\nabla L\left(x_{k+1}, \lambda_{k+1}\right)-\nabla L\left(x_{k}, \lambda_{k+1}\right)\right]-\bar{w}_{k} \\
& =Z_{k}^{T}\left[\int_{0}^{1} \nabla_{x x}^{2} L\left(x_{k}+\tau \alpha_{k} d_{k}, \lambda_{k+1}\right) d \tau\right] \alpha_{k} d_{k}-\bar{w}_{k} \\
& =\alpha_{k} Z_{k}^{T} \tilde{W}_{k}\left(Z_{k} p_{\mathrm{Z}}+Y_{k} p_{\mathrm{Y}}\right)-\bar{w}_{k} \\
& =Z_{k}^{T} \tilde{W}_{k} Z_{k} s_{k}+\alpha_{k}\left(Z_{k}^{T} \tilde{W}_{k}-Z_{*}^{T} W_{*}\right) Y_{k} p_{\mathrm{Y}}+\left(\alpha_{k} Z_{*}^{T} W_{*} Y_{k} p_{\mathrm{Y}}-\bar{w}_{k}\right) .
\end{aligned}
$$

Since $\bar{w}_{k}$ can be computed by Broyden's method or by finite differences, we need to consider these two cases separately. 
Part I. Let us first assume that $\bar{w}_{k}$ is determined by Broyden's method. A simple computation shows that $\left\|Z_{k}^{T} \tilde{W}_{k}-Z_{*}^{T} W_{*}\right\|=O\left(\sigma_{k}\right)$, and from (3.14) we have that $\bar{w}_{k}=O\left(\left\|p_{\mathrm{Y}}\right\| / \gamma_{k}\right)$. Using this and Assumptions 5.1 in (5.27), we have

$$
\begin{aligned}
y_{k} & =Z_{k}^{T} \tilde{W}_{k} Z_{k} s_{k}+\left(\sigma_{k}+1+1 / \gamma_{k}\right) O\left(\alpha_{k}\left\|p_{\mathrm{Y}}\right\|\right) \\
& =\left(Z_{k}^{T} \tilde{W}_{k} Z_{k}-G_{*}\right) s_{k}+G_{*} s_{k}+\left(\sigma_{k}+1+1 / \gamma_{k}\right) O\left(\alpha_{k}\left\|p_{\mathrm{Y}}\right\|\right) .
\end{aligned}
$$

Recalling (5.16) and noting that $\tilde{y}_{k}^{T} \tilde{s}_{k}=y_{k}^{T} s_{k}$, we have

$$
\tilde{y}_{k}^{T} \tilde{s}_{k}=s_{k}^{T}\left(Z_{k}^{T} \tilde{W}_{k} Z_{k}-G_{*}\right) s_{k}+\left\|\tilde{s}_{k}\right\|^{2}+\left(\sigma_{k}+1+1 / \gamma_{k}\right) O\left(\alpha_{k}\left\|p_{\mathrm{Y}}\right\|\right)\left\|\tilde{s}_{k}\right\|,
$$

since $\left\|\tilde{s_{k}}\right\|$ and $\left\|s_{k}\right\|$ are of the same order. Therefore

$$
\begin{aligned}
\frac{\tilde{y}_{k}^{T} \tilde{s}_{k}}{\left\|\tilde{s}_{k}\right\|^{2}} & =1+\frac{s_{k}^{T}\left(Z_{k}^{T} \tilde{W}_{k} Z_{k}-G_{*}\right) s_{k}}{\left\|\tilde{s}_{k}\right\|^{2}}+\left(\sigma_{k}+1+1 / \gamma_{k}\right) O\left(\frac{\left\|\alpha_{k} p_{\mathrm{Y}}\right\|}{\left\|\tilde{s}_{k}\right\|}\right) \\
& =1+O\left(\sigma_{k}\right)+\left(\sigma_{k}+1+1 / \gamma_{k}\right) O\left(\frac{\left\|\alpha_{k} p_{\mathrm{Y}}\right\|}{\left\|\tilde{s}_{k}\right\|}\right) .
\end{aligned}
$$

Similarly from (5.28) and (5.16) we have

$$
\begin{aligned}
\tilde{y}_{k}^{T} \tilde{y}_{k} \leq & \left\|\left(Z_{k}^{T} \tilde{W}_{k} Z_{k}-G_{*}\right) s_{k}\right\|^{2}\left\|G_{*}^{-1}\right\|+2\left\|\left(Z_{k}^{T} \tilde{W}_{k} Z_{k}-G_{*}\right) s_{k}\right\|\left\|G_{*}^{-1 / 2}\right\|\left\|\tilde{s}_{k}\right\|+\left\|\tilde{s}_{k}\right\|^{2} \\
& +2\left(\sigma_{k}+1+1 / \gamma_{k}\right) O\left(\left\|\alpha_{k} p_{\mathrm{Y}}\right\|\right)\left\|G_{*}^{-\frac{1}{2}}\right\|\left(\left\|\tilde{s}_{k}\right\|+\left\|\left(Z_{k}^{T} \tilde{W}_{k} Z_{k}-G_{*}\right) s_{k}\right\|\left\|G_{*}^{-1 / 2}\right\|\right) \\
& +\left(\sigma_{k}+1+1 / \gamma_{k}\right)^{2} O\left(\left\|\alpha_{k} p_{\mathrm{Y}}\right\|\right)^{2},
\end{aligned}
$$

and thus

$$
\begin{aligned}
\frac{\left\|\tilde{y}_{k}\right\|^{2}}{\left\|\tilde{s}_{k}\right\|^{2}} \leq & 1+O\left(\sigma_{k}\right)+\left(\sigma_{k}+1+1 / \gamma_{k}\right)\left(1+\sigma_{k}\right) O\left(\frac{\left\|\alpha_{k} p_{\mathrm{Y}}\right\|}{\left\|\tilde{s}_{k}\right\|}\right) \\
& +\left(\sigma_{k}+1+1 / \gamma_{k}\right)^{2} O\left(\frac{\left\|\alpha_{k} p_{\mathrm{Y}}\right\|^{2}}{\left\|\tilde{s}_{k}\right\|^{2}}\right) .
\end{aligned}
$$

At this point we invoke the update criterion and note from (3.18) that, if BFGS updating of $B_{k}$ takes place at iteration $k$, then $\left\|\alpha_{k} p_{\mathrm{Y}}\right\| \leq \gamma_{k}^{2}\left\|s_{k}\right\|$, where $\left\{\gamma_{k}\right\}$ is summable. Using this, the assumption that $\sigma_{k}$ converges to zero, and (5.29), we see that for large $k$

$$
\frac{\tilde{y}_{k}^{T} \tilde{s}_{k}}{\left\|\tilde{s}_{k}\right\|^{2}}=1+O\left(\sigma_{k}+\gamma_{k}\right)
$$

and using (5.30)

$$
\frac{\left\|\tilde{y}_{k}\right\|^{2}}{\left\|\tilde{s}_{k}\right\|^{2}}=1+O\left(\sigma_{k}+\gamma_{k}\right)
$$

Therefore

$$
\frac{\left\|\tilde{y}_{k}\right\|^{2}}{\tilde{y}_{k}^{T} \tilde{s}_{k}}=\frac{\left\|\tilde{y}_{k}\right\|^{2}}{\left\|\tilde{s}_{k}\right\|^{2}} \frac{\left\|\tilde{s}_{k}\right\|^{2}}{\tilde{y}_{k}^{T} \tilde{s}_{k}}=1+O\left(\sigma_{k}+\gamma_{k}\right) .
$$

We now consider $\psi\left(\tilde{B}_{k+1}\right)$ given by (5.20). A simple expansion shows that for large $k, \ln (1+$ $\left.O\left(\sigma_{k}+\gamma_{k}\right)\right)=O\left(\sigma_{k}+\gamma_{k}\right)$. Using this, (5.31), and (5.32), we have

$$
\psi\left(\tilde{B}_{k+1}\right)=\psi\left(\tilde{B}_{k}\right)+O\left(\sigma_{k}+\gamma_{k}\right)+\ln \cos ^{2} \tilde{\theta}_{k}+\left[1-\frac{\tilde{q}_{k}}{\cos ^{2} \tilde{\theta}_{k}}+\ln \frac{\tilde{q}_{k}}{\cos ^{2} \tilde{\theta}_{k}}\right] .
$$


Note that for $x \geq 0$ the function $1-x+\ln x$ is nonpositive, implying that the term in square brackets is nonpositive and that $\ln \cos ^{2} \tilde{\theta}_{k}$ is also non-positive. We can therefore delete these terms to obtain

$$
\psi\left(\tilde{B}_{k+1}\right) \leq \psi\left(\tilde{B}_{k}\right)+O\left(\sigma_{k}+\gamma_{k}\right) .
$$

Before proceeding further we show that a similar expression holds when finite differences are used.

Part II. Let us now consider the iterates $k$ for which updating takes place and for which $\bar{w}_{k}$ is computed by finite differences. In this case (3.19) holds. Again we begin by considering (5.27),

$$
y_{k}=Z_{k}^{T} \tilde{W}_{k} Z_{k} s_{k}+\alpha_{k}\left(Z_{k}^{T} \tilde{W}_{k}-Z_{*}^{T} W_{*}\right) Y_{k} p_{\mathrm{Y}}+\left(\alpha_{k} Z_{*}^{T} W_{*} Y_{k} p_{\mathrm{Y}}-\bar{w}_{k}\right) .
$$

Using (5.23) the last term is of order $\sigma_{k}\left(\alpha_{k}\left\|p_{\mathrm{Y}}\right\|\right)$, and so is the second term. Thus

$$
\begin{aligned}
y_{k} & =Z_{k}^{T} \tilde{W}_{k} Z_{k} s_{k}+O\left(\sigma_{k} \alpha_{k}\left\|p_{\mathrm{Y}}\right\|\right) \\
& =\left(Z_{k}^{T} \tilde{W}_{k} Z_{k}-G_{*}\right) s_{k}+G_{*} s_{k}+O\left(\sigma_{k} \alpha_{k}\left\|p_{\mathrm{Y}}\right\|\right) .
\end{aligned}
$$

Noting once more that $\tilde{y}_{k}^{T} \tilde{s}_{k}=y_{k}^{T} s_{k}$ and recalling the definition (5.16), we have

$$
\tilde{y}_{k}^{T} \tilde{s}_{k}=s_{k}^{T}\left(Z_{k}^{T} \tilde{W}_{k} Z_{k}-G_{*}\right) s_{k}+\left\|\tilde{s}_{k}\right\|^{2}+O\left(\sigma_{k} \alpha_{k}\left\|p_{\mathrm{Y}}\right\|\left\|\tilde{s}_{k}\right\|\right),
$$

since $\left\|\tilde{s_{k}}\right\|$ and $\left\|s_{k}\right\|$ are of the same order. Therefore

$$
\begin{aligned}
\frac{\tilde{y}_{k}^{T} \tilde{s}_{k}}{\left\|\tilde{s}_{k}\right\|^{2}} & =1+\frac{s_{k}^{T}\left(Z_{k}^{T} \tilde{W}_{k} Z_{k}-G_{*}\right) s_{k}}{\left\|\tilde{s}_{k}\right\|^{2}}+O\left(\sigma_{k} \frac{\left\|\alpha_{k} p_{\mathrm{Y}}\right\|}{\left\|\tilde{s}_{k}\right\|}\right) \\
& =1+O\left(\sigma_{k}\right)+O\left(\sigma_{k} \frac{\left\|\alpha_{k} p_{\mathrm{Y}}\right\|}{\left\|\tilde{s}_{k}\right\|}\right) .
\end{aligned}
$$

Similarly from (5.35) and (5.16) we have

$$
\begin{aligned}
\tilde{y}_{k}^{T} \tilde{y}_{k} \leq & \left\|\left(Z_{k}^{T} \tilde{W}_{k} Z_{k}-G_{*}\right) s_{k}\right\|^{2}\left\|G_{*}^{-1}\right\|+2\left\|\left(Z_{k}^{T} \tilde{W}_{k} Z_{k}-G_{*}\right) s_{k}\right\|\left\|G_{*}^{-1 / 2}\right\|\left\|\tilde{s}_{k}\right\|+\left\|\tilde{s}_{k}\right\|^{2} \\
& +\sigma_{k} O\left(\left\|\alpha_{k} p_{\mathrm{Y}}\right\|\left\|G_{*}^{-\frac{1}{2}}\right\|\left[\left\|\tilde{s}_{k}\right\|+\left\|\left(Z_{k}^{T} \tilde{W}_{k} Z_{k}-G_{*}\right) s_{k}\right\|\left\|G_{*}^{-1 / 2}\right\|\right]\right) \\
& +\sigma_{k}^{2} O\left(\left\|\alpha_{k} p_{\mathrm{Y}}\right\|\right)^{2},
\end{aligned}
$$

and thus

$$
\frac{\left\|\tilde{y}_{k}\right\|^{2}}{\left\|\tilde{s}_{k}\right\|^{2}} \leq 1+O\left(\sigma_{k}\right)+\sigma_{k} O\left(\frac{\left\|\alpha_{k} p_{\mathrm{Y}}\right\|}{\left\|\tilde{s}_{k}\right\|}\right)+\sigma_{k}^{2} O\left(\frac{\left\|\alpha_{k} p_{\mathrm{Y}}\right\|^{2}}{\left\|\tilde{s}_{k}\right\|^{2}}\right) .
$$

We now invoke Update Criterion I and note from (3.19) that, if BFGS updating of $B_{k}$ takes place at iteration $k$, then $\left\|p_{\mathrm{Y}}\right\| \leq \gamma_{\mathrm{fd}}\left\|p_{\mathrm{Z}}\right\| / \sigma_{k}^{1 / 2}$. Using this, (5.36), and the fact that $\sigma_{k}$ converges to zero, we see that for large $k$

$$
\frac{\tilde{y}_{k}^{T} \tilde{s}_{k}}{\left\|\tilde{s}_{k}\right\|^{2}}=1+O\left(\sigma_{k}^{1 / 2}\right),
$$

and using (5.37)

$$
\frac{\left\|\tilde{y}_{k}\right\|^{2}}{\left\|\tilde{s}_{k}\right\|^{2}}=1+O\left(\sigma_{k}^{1 / 2}\right) .
$$

Therefore

$$
\frac{\left\|\tilde{y}_{k}\right\|^{2}}{\tilde{y}_{k}^{T} \tilde{s}_{k}}=\frac{\left\|\tilde{y}_{k}\right\|^{2}}{\left\|\tilde{s}_{k}\right\|^{2}} \frac{\left\|\tilde{s}_{k}\right\|^{2}}{\tilde{y}_{k}^{T} \tilde{s}_{k}}=1+O\left(\sigma_{k}^{1 / 2}\right) .
$$


We now consider $\psi\left(\tilde{B}_{k+1}\right)$ given by (5.20). Noting that $\ln \left(1+O\left(\sigma_{k}^{1 / 2}\right)\right)=O\left(\sigma_{k}^{1 / 2}\right)$ for all large $k$, we see that if updating takes place at iteration $k$

$$
\psi\left(\tilde{B}_{k+1}\right)=\psi\left(\tilde{B}_{k}\right)+O\left(\sigma_{k}^{1 / 2}\right)+\ln \cos ^{2} \tilde{\theta}_{k}+\left[1-\frac{\tilde{q}_{k}}{\cos ^{2} \tilde{\theta}_{k}}+\ln \frac{\tilde{q}_{k}}{\cos ^{2} \tilde{\theta}_{k}}\right] .
$$

Since both $\ln \cos ^{2} \tilde{\theta}_{k}$ and the term inside the square brackets are nonpositive, we can delete them to obtain

$$
\psi\left(\tilde{B}_{k+1}\right) \leq \psi\left(\tilde{B}_{k}\right)+O\left(\sigma_{k}^{1 / 2}\right) .
$$

We now combine the results of Parts I and II of this proof. Let us subdivide the set of iterates $U$ for which BFGS updating takes place into two subsets: $U^{\prime}$ corresponds to the iterates in which $\bar{w}_{k}$ is computed by Broyden's method, and $U^{\prime \prime}$ to the iterates in which finite differences are used. We also define $U_{k}^{\prime}=U^{\prime} \cap\{1,2, \ldots, k\}$ and $U_{k}^{\prime \prime}=U^{\prime \prime} \cap\{1,2, \ldots, k\}$.

Summing over the set of iterates in $U_{k}$, using (5.34) and (5.40), and noting that $B_{j+1}=B_{j}$ for $j \notin U_{k}$, we have

$$
\psi\left(\tilde{B}_{k+1}\right) \leq \psi\left(\tilde{B}_{1}\right)+C_{1} \sum_{j \in U_{k}^{\prime \prime}} \sigma_{j}^{1 / 2}+C_{2} \sum_{j \in U_{k}^{\prime \prime}} \sigma_{j}+C_{3} \sum_{j \in U_{k}^{\prime}} \gamma_{j},
$$

for some constants $C_{1}, C_{2}, C_{3}$. Since $0 \leq r \leq 1$ and $\left|U_{j}^{\prime \prime}\right| \leq\left|U_{j}\right|$ we have, from (5.11)

$$
\begin{aligned}
\sum_{j \in U^{\prime \prime}} \sigma_{j}^{1 / 2} & \leq \sum_{j \in U^{\prime \prime}} C^{1 / 2} r^{\left|U_{j}\right| / 2} \\
& \leq \sum_{j \in U^{\prime \prime}} C^{1 / 2} r^{\left|U_{j}^{\prime \prime}\right| / 2} \\
& =\sum_{i=1}^{\left|U^{\prime \prime}\right|} C^{1 / 2} r^{i / 2} \\
& <\infty .
\end{aligned}
$$

Similarly

$$
\sum_{j \in U^{\prime}} \sigma_{j}<\infty
$$

and since $\left\{\gamma_{k}\right\}$ is summable, we conclude from (5.41) that $\left\{\psi\left(\tilde{B}_{k}\right)\right\}$ is bounded above. By (5.14) $\psi\left(\tilde{B}_{k}\right)=\sum_{i=1}^{n}\left(l_{i}-\ln l_{i}\right)$, where $l_{i}$ are the eigenvalues of $\tilde{B}_{k}$, and it is easy to see that this implies that both $\left\|B_{k}\right\|$ and $\left\|B_{k}^{-1}\right\|$ are bounded.

To prove (5.26), we sum relations (5.33) and (5.39), recalling that $\sigma_{k}, \gamma_{k}$ and $\sigma_{k}^{1 / 2}$ are summable, to obtain

$$
\psi\left(\tilde{B}_{k+1}\right) \leq C+\sum_{j \in U_{k}}\left(\ln \cos ^{2} \tilde{\theta}_{k}+\left[1-\frac{\tilde{q}_{k}}{\cos ^{2} \tilde{\theta}_{k}}+\ln \frac{\tilde{q}_{k}}{\cos ^{2} \tilde{\theta}_{k}}\right]\right),
$$

for some constant $C$. Since $\psi\left(\tilde{B}_{k+1}\right)>0$, and since both $\ln \cos ^{2} \theta_{k}$ and the term inside the square brackets are nonpositive, we see that

$$
\lim _{\substack{k \rightarrow \infty \\ k \in U}} \ln \cos ^{2} \tilde{\theta}_{k}=0
$$


and

$$
\lim _{\substack{k \rightarrow \infty \\ k \in U}}\left[1-\frac{\tilde{q}_{k}}{\cos ^{2} \tilde{\theta}_{k}}+\ln \frac{\tilde{q}_{k}}{\cos ^{2} \tilde{\theta}_{k}}\right] \rightarrow 0 .
$$

Now, for $x \geq 0$ the function $1-x+\ln x$ is concave and has its unique maximizer at $x=1$. Therefore the relations above imply that

$$
\lim _{\substack{k \rightarrow \infty \\ k \in U}} \cos \tilde{\theta}_{k}=\lim _{\substack{k \rightarrow \infty \\ k \in U}} \tilde{q}_{k}=1 .
$$

Now from (5.18)-(5.19)

$$
\begin{aligned}
\frac{\left\|G_{*}^{-1 / 2}\left(B_{k}-G_{*}\right) p_{z}\right\|^{2}}{\left\|G_{*}^{1 / 2} p_{\mathrm{Z}}\right\|^{2}} & =\frac{\left\|\left(\tilde{B}_{k}-I\right) \tilde{s}_{k}\right\|^{2}}{\left\|\tilde{s}_{k}\right\|^{2}} \\
& =\frac{\left\|\tilde{B}_{k} \tilde{s}_{k}\right\|^{2}-2 \tilde{s}_{k}^{T} \tilde{B}_{k} \tilde{s}_{k}+\tilde{s}_{k}^{T} \tilde{s}_{k}}{\tilde{s}_{k}^{T} \tilde{s}_{k}} \\
& =\frac{\tilde{q}_{k}^{2}}{\cos \tilde{\theta}_{k}^{2}}-2 \tilde{q}_{k}+1 .
\end{aligned}
$$

It is clear from (5.42) that the last term converges to 0 for $k \in U$, which implies that (5.26) holds.

This result immediately implies that the iterates are R-linearly convergent, regardless of how often updating takes place.

Theorem 5.7 Suppose that the iterates $\left\{x_{k}\right\}$ generated by Algorithm II converge to a solution point $x_{*}$ that satisfies Assumptions 5.1 and the fact that $|U| \rightarrow \infty$. Then the rate of convergence is at least R-linear.

Proof. Theorem 5.6 implies that the condition number of the matrices $\left\{B_{k}\right\}$ is bounded. Therefore, all the iterates are good iterates. Reasoning as in the proof of Theorem 5.4, we conclude that for all $j$

$$
\left\|x_{j}-x_{*}\right\| \leq C r^{j}
$$

for some constants $C>0$ and $0 \leq r<1$.

Prior to considering the convergence rate, we show that the Broyden matrices $S_{k}$ are bounded.

Lemma 5.8 Suppose that the iterates $\left\{x_{k}\right\}$ generated by Algorithm II converge R-linearly to a solution point $x_{*}$ that satisfies Assumptions 5.1. Then the Broyden matrices $S_{k}$ are bounded and the safeguards (3.13) and (3.14) become inactive for all large $k$.

Proof. We make use of the well-known bounded deterioration property for Broyden's method (cf. Lemma 8.2.1 in Dennis and Schnabel (1983)), which states that under Assumptions 5.1

$$
\left\|S_{k+1}-Z_{*}^{T} W_{*}\right\| \leq\left\|S_{k}-Z_{*}^{T} W_{*}\right\|+C \sigma_{k},
$$

for some constant $C>0$. As a result of the R-linear convergence of $\left\{x_{k}\right\}$, we obtain

$$
\begin{aligned}
\left\|S_{k+1}-Z_{*}^{T} W_{*}\right\| & \leq\left\|S_{1}-Z_{*}^{T} W_{*}\right\|+C \sum_{i=1}^{k} \sigma_{k} \\
& <\infty
\end{aligned}
$$


which shows that the matrices $S_{k}$ remain bounded. We then see from (3.12) that the Broyden corrections $w_{k}$ and $\bar{w}_{k}$ satisfy

$$
w_{k}=O\left(\left\|p_{\mathrm{Y}}\right\|\right) \quad \bar{w}_{k}=O\left(\left\|p_{\mathrm{Y}}\right\|\right)
$$

and it is clear that the safeguards (3.13) and (3.14) become inactive for all large $k$.

Therefore, the algorithm will not modify the information supplied by Broyden's method, asymptotically. This is an important point in establishing superlinear convergence.

\section{Superlinear Convergence}

Without the correction terms $w_{k}$ and $\bar{w}_{k}$, and with appropriate update criteria, Algorithm II is 2-step Q-superlinearly convergent. This was proved by Nocedal and Overton (1985) assuming that $Y_{k}$ and $Z_{k}$ are orthogonal bases and assuming that a good starting matrix $B_{1}$ is used. This result has been extended by Xie (1991) for more general bases and for any starting matrix $B_{1}>0$. In this section we will show that if the correction terms are used in Algorithm II, the rate of convergence is 1-step Q-superlinear. This result is possible by Update Criterion I and by the selected application of finite-difference approximations, which allow BFGS updating to occur more frequently.

To establish superlinear convergence, we need to ensure that the steplengths $\alpha_{k}$ have the value 1 for all large $k$. When a smooth merit function, such as Fletcher's differentiable function (Fletcher (1973)) is used, it is not difficult to show that, near the solution, unit steplengths give a sufficient reduction in the merit function and will be accepted. However, the nondifferentiable $\ell_{1}$ merit function (1.7) used in this paper may reject steplengths of one, even very close to the solution. This so-called Maratos effect requires that the algorithm be modified to allow unit steplengths and to achieve a fast rate of convergence. We will not consider this modification here, so as not to complicate our already lengthy analysis and since it does not affect the main structure of the algorithm or its essential properties. In the companion paper (Biegler, Nocedal, and Schmid (1993)), which is devoted to a numerical investigation of Algorithm II, we describe how to incorporate the nonmonotone line search (or watchdog technique) of Chamberlain et al. (1982) that allows unit steplengths to be accepted for all large $k$. The analysis of the modified algorithm would be similar to that presented in $\S 5.5$ of Byrd and Nocedal (1991).

In the remainder of this section we assume that the iterates generated by Algorithm II converge R-linearly to a solution and that unit steplengths are taken for all large $k$. In the presentation of the results that follow we do not restate the assumptions under which R-linear convergence was proved in $\S 5$, but simply assume that R-linear convergence occurs. We begin by showing that the damping parameter $\zeta_{k}$, used in (3.43) to ensure that descent directions are always generated, has the value of 1 for all large $k$.

We have shown in Theorem 5.6 that $\left\|B_{k}^{-1}\right\|$ is bounded above. Also, (5.21), (5.2), and (3.42) show that, when finite differences are used, $w_{k}=O\left(\left\|p_{\mathrm{Y}}\right\|\right)=O\left(\left\|c_{k}\right\|\right)$, and by (5.43) we see that this is also the case when Broyden's method is used. Using these facts, and noting that $\|\cdot\| \leq\|\cdot\|_{1}$, we see that there is a constant $C$ such that the left-hand side of (3.38) can be bounded by

$$
\zeta_{k}\left[2 \cos \theta_{k}\left|g_{k}^{T} Z_{k} w_{k}\right|+w_{k}^{T} B_{k}^{-1} Z_{k}^{T} g_{k}+\zeta_{k} w_{k}^{T} B_{k}^{-1} w_{k}\right] \leq\left[\zeta_{k} C\left(\left\|e_{k}\right\|+\zeta_{k}\left\|c_{k}\right\|\right)\right]\left\|c_{k}\right\|_{1},
$$

since $g_{k}^{T} Z_{k}=O\left(\left\|e_{k}\right\|\right)$. As the iterates converge to the solution, and since $\zeta_{k} \leq 1$, the term inside the square brackets is less than the constant $\rho$ given in (3.38), showing that $\zeta_{k}=1$ for all large $k$. This and the remarks made at the end of $\oint 5$ show that all the safeguards included in Algorithm II become inactive asymptotically. 
We can now show that the Broyden matrices satisfy the condition of Dennis and Moré (1974) for superlinear convergence. Note from Algorithm II that a Broyden update of $S_{k}$ is always performed, regardless of whether a BFGS update of $B_{k}$ takes place or not. The following result is a straightforward modification of a well-known property for Broyden's method.

Lemma 6.1 Suppose that the iterates generated by Algorithm II converge R-linearly to a point $x_{*}$ that satisfies Assumptions 5.1. Then

$$
\lim _{k \rightarrow \infty} \frac{\left\|\left(S_{k}-Z_{*}^{T} W_{*}\right) d_{k}\right\|}{\left\|d_{k}\right\|}=0 .
$$

Proof. The proof is essentially given in Griewank (1986) and is also very similar to the analysis in Dennis and Schnabel (1983, pp. 183-4), but we will give it here for the sake of completeness. Using the Broyden formula (3.9), we have

$$
\begin{aligned}
S_{k+1}-Z_{*}^{T} W_{*} & =S_{k}-Z_{*}^{T} W_{*}+\frac{\left(\bar{y}_{k}-S_{k} \bar{s}_{k}\right) \bar{s}_{k}^{T}}{\bar{s}_{k}^{T} \bar{s}_{k}} \\
& =S_{k}-Z_{*}^{T} W_{*}+\frac{\left(\bar{y}_{k}-Z_{*}^{T} W_{*} \bar{s}_{k}\right) \bar{s}_{k}^{T}}{\bar{s}_{k}^{T} \bar{s}_{k}}+\frac{\left(Z_{*}^{T} W_{*}-S_{k}\right) \bar{s}_{k} \bar{s}_{k}^{T}}{\bar{s}_{k}^{T} \bar{s}_{k}} \\
& =\left(S_{k}-Z_{*}^{T} W_{*}\right)\left(I-\bar{s}_{k} \bar{s}_{k}^{T} / \bar{s}_{k}^{T} \bar{s}_{k}\right)+\left(\bar{y}_{k}-Z_{*}^{T} W_{*} \bar{s}_{k}\right) \bar{s}_{k}^{T} / \bar{s}_{k}^{T} \bar{s}_{k} .
\end{aligned}
$$

Defining $E_{k}=S_{k}-Z_{*}^{T} W_{*}$, applying Lemma 8.2.5 of Dennis and Schnabel (1983), recalling (3.10)-(3.11), and using the Mean Value theorem, we obtain

$$
\begin{aligned}
\left\|E_{k+1}\right\|_{F} & \leq\left\|E_{k}\left(I-\bar{s}_{k} \bar{s}_{k}^{T} / \bar{s}_{k}^{T} \bar{s}_{k}\right)\right\|_{F}+O\left(\sigma_{k}\right) \\
& \leq\left\|E_{k}\right\|_{F}-\frac{\left\|E_{k} \bar{s}_{k}\right\|^{2}}{2\left\|E_{k}\right\|_{F}\left\|\bar{s}_{k}\right\|^{2}}+O\left(\sigma_{k}\right) .
\end{aligned}
$$

Rearranging this expression yields

$$
\frac{\left\|E_{k} \bar{s}_{k}\right\|^{2}}{\left\|\bar{s}_{k}\right\|^{2}} \leq 2\left\|E_{k}\right\|_{F}\left[\left\|E_{k}\right\|_{F}-\left\|E_{k+1}\right\|_{F}+O\left(\sigma_{k}\right)\right] .
$$

By Lemma 5.8 , we know that the matrices $S_{k}$ remain bounded, therefore there exists some $\Delta$ such that for all $k \geq \bar{k},\left\|E_{k}\right\| \leq \Delta / 2$ and

$$
\sum_{k=\bar{k}}^{\infty} \frac{\left\|E_{k} \bar{s}_{k}\right\|^{2}}{\left\|\bar{s}_{k}\right\|^{2}} \leq \Delta\left[\left\|E_{\bar{k}}\right\|_{F}+\sum_{k=\bar{k}}^{\infty} O\left(\sigma_{k}\right)\right] .
$$

Since $\left\{\sigma_{k}\right\}$ converges R-linearly, the last term is summable, which implies that

$$
\lim _{k \rightarrow \infty} \frac{\left\|E_{k} \bar{s}_{k}\right\|^{2}}{\left\|\bar{s}_{k}\right\|^{2}}=0 .
$$

Noting that $\bar{s}_{k}=\alpha_{k} d_{k}$ gives the desired result.

This lemma shows that in the limit $S_{k}$ is an accurate approximation to $Z_{*}^{T} W_{*}$ along $d_{k}$, and Theorem 5.6 shows that, when updating takes place, $B_{k}$ is an accurate approximation to $Z_{*}^{T} W_{*} Z_{*}$ along $p_{\mathrm{Y}}$. We will use these two facts and the following lemma, which is an application of the well-known result of Boggs, Tolle, and Wang (1982). 
Lemma 6.2 Suppose that the iterates generated by Algorithm II converge R-linearly to a point $x_{*}$ that satisfies Assumptions 5.1, and suppose that $\alpha_{k}=1$ for all large $k$. If, in addition,

$$
\lim _{k \rightarrow \infty} \frac{\left\|B_{k} p_{\mathrm{Z}}+w_{k}-Z_{*}^{T} W_{*} d_{k}\right\|}{\left\|d_{k}\right\|}=0,
$$

then the rate of convergence is 1-step Q-superlinear.

Proof. Nocedal and Overton (1985, Theorem 3.2) show that if an algorithm of the form

$$
\begin{gathered}
{\left[\begin{array}{c}
\tilde{S}_{k} \\
A_{k}^{T}
\end{array}\right] d_{k}=-\left[\begin{array}{c}
Z_{k}^{T} g_{k} \\
c_{k}
\end{array}\right],} \\
x_{k+1}=x_{k}+d_{k},
\end{gathered}
$$

converges to a point $x_{*}$ that satisfies Assumptions 5.1, and if

$$
\lim _{k \rightarrow \infty} \frac{\left\|\left(\tilde{S}_{k}-Z_{*}^{T} W_{*}\right) d_{k}\right\|}{\left\|d_{k}\right\|}=0
$$

then the rate of convergence is superlinear. Algorithm II clearly satisfies the second equation in (6.4), $A_{k}^{T} d_{k}=-c_{k}$. Now, since $d_{k}=Y_{k} p_{\mathrm{Y}}+Z_{k} p_{\mathrm{Z}}$, we have

$$
\left[Y_{k} Z_{k}\right]^{-1} d_{k}=\left[\begin{array}{l}
p_{\mathrm{Y}} \\
p_{\mathrm{Z}}
\end{array}\right]
$$

Let us write $w_{k}=T_{k} p_{\mathrm{Y}}$ for some matrix $T_{k}$. Then, recalling that $\zeta_{k}=1$ for all large $k$, we have from (3.43) that

$$
\left[\begin{array}{ll}
T_{k} & B_{k}
\end{array}\right]\left[Y_{k} Z_{k}\right]^{-1} d_{k}=-Z_{k}^{T} g_{k} .
$$

Thus we can define $\tilde{S}_{k}=\left[T_{k} B_{k}\right]\left[Y_{k} Z_{k}\right]^{-1}$, and the condition (6.5) for superlinear convergence is

$$
\lim _{k \rightarrow \infty} \frac{\left\|\left(\left[T_{k} B_{k}\right]\left[Y_{k} Z_{k}\right]^{-1}-Z_{*}^{T} W_{*}\right) d_{k}\right\|}{\left\|d_{k}\right\|}=0
$$

However, using (6.6) and $w_{k}=T_{k} p_{\mathrm{Y}}$, we have that $\left[T_{k} B_{k}\right]\left[Y_{k} Z_{k}\right]^{-1} d_{k}=T_{k} p_{\mathrm{Y}}+B_{k} p_{\mathrm{Z}}=w_{k}+B_{k} p_{\mathrm{Z}}$, giving the desired result.

We can now prove the final result of this section. The analysis is complicated by the fact that BFGS updating may not always take place, and by the fact that the correction terms are sometimes computed by finite differences and sometimes by Broyden's method. We therefore consider the following three sets of iterates, based on Update Criterion I and illustrated in Figure 2.

- $R_{1}=\left\{j \mid\left\|p_{\mathrm{Y}}^{(j)}\right\| \leq \gamma_{j}^{2}\left\|p_{\mathrm{Z}}^{(j)}\right\|\right\}$,

- $R_{2}=\left\{j \notin R_{1} \mid\left\|p_{\mathrm{Y}}^{(j)}\right\| \leq\left\|p_{\mathrm{Z}}^{(j)}\right\| / \sigma_{j}^{1 / 2}\right\}$,

- $R_{3}=\left\{j\left\|p_{\mathrm{Y}}^{(j)}\right\|>\left\|p_{\mathrm{Z}}^{(j)}\right\| / \sigma_{j}^{1 / 2}\right\}$,

and note that both $\gamma_{k}$ and $\sigma_{k}$ are summable.

Theorem 6.3 Suppose that the iterates generated by Algorithm II converge R-linearly to a point $x_{*}$ that satisfies Assumptions 5.1, and suppose that $\alpha_{k}=1$ for all large $k$. Then the rate of convergence is 1-step Q-superlinear. 
Proof. Since $d_{k}=Y_{k} p_{\mathrm{Y}}+Z_{k} p_{\mathrm{Z}}$, we have

$$
\left[\begin{array}{c}
p_{\mathrm{Y}} \\
p_{\mathrm{Z}}
\end{array}\right]=\left[Y_{k} Z_{k}\right]^{-1} d_{k}
$$

Therefore, Assumption (5.3) implies that

$$
\left\|p_{\mathrm{Y}}\right\|=O\left(\left\|d_{k}\right\|\right), \quad\left\|p_{\mathrm{Z}}\right\|=O\left(\left\|d_{k}\right\|\right) .
$$

Now

$$
\begin{aligned}
\left\|B_{k} p_{\mathrm{Z}}+w_{k}-Z_{*}^{T} W_{*} d_{k}\right\| \leq & \left\|B_{k} p_{\mathrm{Z}}-Z_{*}^{T} W_{*} Z_{k} p_{\mathrm{Z}}\right\|+\left\|w_{k}-Z_{*}^{T} W_{*} Y_{k} p_{\mathrm{Y}}\right\| \\
\leq & \left\|B_{k} p_{\mathrm{Z}}-Z_{*}^{T} W_{*} Z_{*} p_{\mathrm{Z}}\right\|+\left\|w_{k}-Z_{*}^{T} W_{*} Y_{k} p_{\mathrm{Y}}\right\| \\
& +O\left(\left\|e_{k}\right\|\left\|p_{\mathrm{Z}}\right\|\right) .
\end{aligned}
$$

Since by (6.7) the last term is of order $o\left(\left\|p_{Z}\right\|\right)=o\left(\left\|d_{k}\right\|\right)$, the objective of the proof is to show that

$$
\left\|B_{k} p_{\mathrm{Z}}-Z_{*}^{T} W_{*} Z_{*} p_{\mathrm{Z}}\right\|+\left\|w_{k}-Z_{*}^{T} W_{*} Y_{k} p_{\mathrm{Y}}\right\|=o\left(\left\|d_{k}\right\|\right),
$$

for this together with (6.3) will give the desired result. We consider the three regions $R_{1}, R_{2}$, and $R_{3}$ separately. Algorithm II is designed so that, in $R_{2}, w_{k}$ must be computed by finite differences. On the other hand, since $p_{\mathrm{Z}}$ is recomputed in Step 7, after which we can be in any of the three regions, we see that in $R_{1}$ and $R_{3} w_{k}$ may be computed by finite differences or by Broyden.

If $k \in R_{1}$, we have that $\left\|p_{\mathrm{Y}}\right\|=o\left(\left\|p_{\mathrm{Z}}\right\|\right)=o\left(\left\|d_{k}\right\|\right)$. We also know from (5.43) that $w_{k}=$ $O\left(\left\|p_{\mathrm{Y}}\right\|\right)$ when the correction is computed by Broyden's method, and by (5.21) this relation also holds when $w_{k}$ is computed by finite differences. Therefore, for $k \in R_{1}$,

$$
\left\|w_{k}-Z_{*}^{T} W_{*} Y_{k} p_{\mathrm{Y}}\right\|=o\left(\left\|d_{k}\right\|\right) .
$$

Furthermore, since updating always takes place in $R_{1},(5.26)$ holds:

$$
\left\|B_{k} p_{\mathrm{Z}}-Z_{*}^{T} W_{*} Z_{*} p_{\mathrm{Z}}\right\|=o\left(\left\|d_{k}\right\|\right) .
$$

We have thus established (6.8) for all $k \in R_{1}$.

Let us now suppose that $k \in R_{2}$, in which case $w_{k}$ is computed by finite differences. Using (5.22), we have that

$$
\left\|w_{k}-Z_{*}^{T} W_{*} Y_{k} p_{\mathrm{Y}}\right\|=o\left(\left\|p_{\mathrm{Y}}\right\|\right)=o\left(\left\|d_{k}\right\|\right),
$$

where the last step follows from (6.7). Since updating always takes place in $R_{2}$, Equation (6.10) also holds in this case, and we conclude that (6.8) holds for all $k \in R_{2}$.

Finally we consider the case when $k \in R_{3}$. Now $p_{\mathrm{Z}}$ satisfies

$$
p_{\mathrm{Z}}=o\left(\left\|p_{\mathrm{Y}}\right\|\right)=o\left(\left\|d_{k}\right\|\right) .
$$

If $k \in R_{3}$ and the correction term $w_{k}$ is computed by Broyden's method as $w_{k}=S_{k} Y_{k} p_{\mathrm{Y}}$ (see (3.12)), we have

$$
\begin{aligned}
\left\|w_{k}-Z_{*}^{T} W_{*} Y_{k} p_{\mathrm{Y}}\right\| & =\left\|\left(S_{k}-Z_{*}^{T} W_{*}\right) Y_{k} p_{\mathrm{Y}}\right\| \\
& \leq\left\|\left(S_{k}-Z_{*}^{T} W_{*}\right) d_{k}\right\|+\left\|\left(S_{k}-Z_{*}^{T} W_{*}\right) Z_{k} p_{\mathrm{Z}}\right\| .
\end{aligned}
$$

Using (6.1), (6.12), and the boundedness of $S_{k}$, we see that the right-hand side is of order $o\left(\left\|d_{k}\right\|\right)$, so that $(6.11)$ holds. On the other hand, if $w_{k}$ is computed by finite differences, we have directly 
from (5.22) that (6.11) holds. In addition, (6.12) and the boundedness of $B_{k}$ show that (6.10) holds for all $k \in R_{3}$, regardless of whether finite differences or Broyden's method are used.

\section{Final Remarks}

We have presented a new reduced Hessian algorithm for large-scale equality-constrained optimization. The motivation for this work has been practical: our earlier reduced Hessian code, designed for large problems, was often subject to instabilities, and we have aimed to develop a more robust algorithm that resembles the full-space SQP method but is less expensive to implement. In a forthcoming paper (Biegler, Nocedal, and Schmid (1993)), we discuss our computational experience with the new method. That paper describes how to handle inequality constraints and discusses numerous important details of implementation not considered here. These include the choices of all constants and tolerances, the strategy for coping with the case when the basis matrix $C$ in (2.1) changes, and the procedure for computing the damping parameter $\zeta_{k}$, which was only outlined in (3.39). We also discuss in that paper how to apply the updating criterion away from the solution. We believe that the new algorithm can be very useful for solving large problems, especially those with few degrees of freedom.

We have focused only on convergence results that helped us in the design of the algorithm and that revealed its main properties. The analysis was complicated by two factors. We did not assume that the BFGS matrices $B_{k}$ or the Broyden matrices $S_{k}$ were bounded, which required careful consideration of their behavior. This analysis paid off by suggesting safeguards that are useful in practice and ensure a superlinear rate of convergence. The other complicating factor was the fact that the frequency of BFGS updating can vary drastically: it can take place at every iteration, never, or in various patterns. As was found earlier by Xie (1991), it is necessary to develop the theory in sufficient generality to cover all of these cases, and this significantly increased the complexity of some of the results.

Acknowledgments. We thank R. Byrd for many interesting discussions on the subject of this paper. We are also thankful to a referee who made very useful suggestions on how to improve the presentation of the results. 
8. *

References

[1] L. T. Biegler, J. Nocedal, And C. Schmid, in preparation, 1993.

[2] P. T. Boggs, J. W. Tolle, And P. Wang, On the local convergence of a quasiNewton method for constrained optimization, SIAM J. Control Optim., 20 (1982), pp. 161-171.

[3] R. H. BYRD, An example of irregular convergence in some constrained optimization methods that use the projected Hessian, Math. Programming, 32 (1985), pp. 232-237.

[4] R. H. BYRD, On the convergence of constrained optimization methods with accurate Hessian information on a subspace, SIAM J. Numer. Anal., 27 (1990), pp. 141-153.

[5] R. H. Byrd and J. Nocedal, A tool for the analysis of quasi-Newton methods with application to unconstrained minimization, SIAM J. Numer. Anal., 26 (1989), pp. $727-739$.

[6] R. H. Byrd And J. Nocedal, An analysis of reduced Hessian methods for constrained optimization, Math. Programming, 49 (1991), pp. 285-323.

[7] R. H. Byrd, J. Nocedal, And R. B. Schnabel, Representations of quasi-Newton matrices and their application to limited memory methods, to appear in Mathematical Programming, 1992.

[8] R. H. Byrd, J. Nocedal, ANd Y. YuAn, Global convergence of a class of quasiNewton methods on convex problems, SIAM J. Numer. Anal., 24 (1987), pp. 11711190.

[9] R. M. Chamberlain, C. Lemarechal, H. C. Pedersen, and M. J. D. Powell, The watchdog technique for forcing convergence in algorithms for constrained optimization, Math. Programming Studies, 16 (1982), pp. 1-17.

[10] T. F. Coleman And A. R. Conn, Nonlinear programming via an exact penalty function: global analysis, Math. Programming, 24 (1982), pp. 137-161.

[11] T. F. Coleman And A. R. Conn, On the local convergence of a quasi-Newton method for the nonlinear programming problem, SIAM J. Numer. Anal., 21 (1984), pp. 755769 .

[12] T. F. Coleman And P. A. Fenyes, Partitioned quasi-Newton methods for nonlinear equality constrained optimization, Math. Programming, 53 (1992), pp. 17-44.

[13] A. R. Conn, Constrained optimization using a nondifferentiable penalty function, SIAM J. Numer. Anal., 13 (1973), pp. 145-154.

[14] J. E. Dennis And J. J. Moré, A characterization of superlinear convergence and its application to quasi-Newton methods, Math. Comp., 28 (1974), pp. 549-560.

[15] J. E. Dennis, JR. And R. B. Schnabel, Numerical Methods for Unconstrained Optimization and Nonlinear Equations, Prentice-Hall, Inc., Englewood Cliffs, N.J., 1983.

[16] R. Fletcher, An exact penalty for nonlinear programming with inequalities, Math. Programming, 5 (1973), pp. 129-150. 
[17] R. Fletcher, Practical Methods of Optimization (second edition), John Wiley and Sons, Chichester, 1987.

[18] D. GABAY, Reduced quasi-Newton methods with feasibility improvement for nonlinearly constrained optimization, Math. Programming Studies, 16 (1982), pp. 18-44.

[19] J. C. GilBerT, On the local and global convergence of a reduced quasi-Newton method, Optimization, 20 (1989), pp. 421-450.

[20] J. C. Gilbert, Maintaining the positive definiteness of the matrices in reduced Hessian methods for equality constrained optimization, Math. Programming, 50 (1991), pp. $1-28$.

[21] A. Griewank, The "global" convergence of Broyden-like methods with a suitable line search, J. Austral. Math. Soc. Ser. B 28 (1986), pp. 75-92.

[22] C. B. Gurwitz, Local convergence of a two-piece update of a projected Hessian matrix, Tech. Report, Department of Computer and Information Science, Brooklyn College, to appear in SIAM J. Optimization, 1993.

[23] C. B. Gurwitz and M. L. Overton, SQP methods based on approximating a projected Hessian matrix, SIAM. J. Sci. Stat. Comp., 10 (1989), pp. 631-653.

[24] S. P. HAN, A globally convergent method for nonlinear programming, J. Optimization Theory and Application, 22/3 (1977), pp. 297-309.

[25] W. Murray and F. J. Prieto, A sequential quadratic programming algorithm using an incomplete solution of the subproblem, Tech Report, Department of Operations Research, Stanford University, (1992).

[26] J. Nocedal and M. L. Overton, Projected Hessian updating algorithms for nonlinearly constrained optimization, SIAM J. Numer. Anal., 22 (1985), pp. 821-850.

[27] M. J. D. PowelL, The convergence of variable metric methods for nonlinearly constrained optimization calculations, in: O. Mangasarian, R. Meyer, and S. Robinson, eds., Nonlinear Programming, 3, pp. 27-63, Academic Press, New York, 1978.

[28] F. TAgliaferro, On a quasi-Newton update and its application to equality constrained optimization, Technical Report, Universita di Trieste, Italy, 1989.

[29] Y. XIE, Reduced Hessian algorithms for solving large-scale equality constrained optimization problems, Ph.D. dissertation, Department of Computer Science, University of Colorado, Boulder, 1991.

[30] Y. YUAN, An only 2-step Q-superlinear convergence example for some algorithms that use reduced Hessian approximations, Math. Programming, 32 (1985), pp. 224231. 